TRANSACTIONS OF THE

AMERICAN MATHEMATICAL SOCIETY

Volume 357, Number 1, Pages 221-245

S 0002-9947(04)03694-3

Article electronically published on August 19, 2004

\title{
ELEMENTS OF SPECIFIED ORDER IN SIMPLE ALGEBRAIC GROUPS
}

\author{
R. LAWTHER
}

\begin{abstract}
In this paper we let $G$ be a simple algebraic group and $r$ be a natural number, and consider the codimension in $G$ of the variety of elements $g \in G$ satisfying $g^{r}=1$. We shall obtain a lower bound for this codimension which is independent of characteristic, and show that it is attained if $G$ is of adjoint type.
\end{abstract}

Let $G$ be a simple algebraic group over an algebraically closed field $K$ of characteristic $p$; let $\Phi$ be the root system of $G$, and take $r \in \mathbb{N}$. Define

$$
G_{[r]}=\left\{g \in G: g^{r}=1\right\} \quad \text { and } \quad G_{(r)}=\{g \in G: o(g)=r\}
$$

(where $o(g)$ denotes the order of $g$ ); then $G_{[r]}$ and $G_{(r)}$ are both subvarieties of $G$, and $G_{[r]}$ is the disjoint union of those $G_{\left(r^{\prime}\right)}$ with $r^{\prime}$ dividing $r$. Our attention here is on the codimension in $G$ of these varieties (if they are non-empty; clearly $G_{[r]} \neq \emptyset$, but the example of $G=S L_{2}(K), p=2$ and $r=4$ shows that $G_{(r)}$ may be empty). It is immediate that if $G_{(r)} \neq \emptyset$ we have $\operatorname{codim} G_{(r)} \geq \operatorname{codim} G_{[r]}$. Our main result may be stated as follows.

Theorem 1. Given $G, \Phi$ and $r$ as above, there is a number $d_{\Phi, r}$, depending only on $\Phi$ and $r$ and satisfying $d_{\Phi, r} \geq|\Phi| / r$, with the property that codim $G_{[r]} \geq d_{\Phi, r}$; if $G$ is of adjoint type we in fact have $\operatorname{codim} G_{[r]}=d_{\Phi, r}$, and if in addition $G_{(r)} \neq \emptyset$, then $\operatorname{codim} G_{(r)}=d_{\Phi, r}$.

Statements equivalent to the inequality $\operatorname{codim} G_{(r)} \geq|\Phi| / r$ are already known in certain cases. If $r=2$ and $p \neq 2$, the equivalent statement that, if $g \in G$ is an involution, then $\operatorname{dim} C_{G}(g) \geq \operatorname{dim}(G / B)$ (where $B$ is a Borel subgroup), is well known; the stronger statement that $C_{G}(g)$ is then spherical, i.e., it has finitely many orbits on the flag variety $G / B$, was proved by Matsuki in 13 for $K=\mathbb{C}$, and by Springer in 19] for $p$ odd - recently Seitz gave an alternative proof of Springer's result in [16. In the case $r=3$ and $p \neq 3$, the result follows from work of Liebeck and Shalev in [10; this case and that with $r=2$ are used in work of Liebeck, Seitz and the author concerning dimensions of fixed point spaces in [9]. More generally, for $r$ an odd prime a result in this direction appears in further work of Liebeck and Shalev in [11, while the results proved here find application in [12] to homomorphisms from Fuchsian groups to finite simple groups.

Notice in particular that the statement of Theorem 1 is independent of the characteristic $p$. The author is grateful to Martin Liebeck for the initial observation

Received by the editors July 18, 2003.

2000 Mathematics Subject Classification. Primary 20G15.

(C) 2004 American Mathematical Society Reverts to public domain 28 years from publication 
that there appeared to be independence of $p$ for $r$ small and prime, which helped to motivate the present work.

Some calculations similar to certain ones here appear in a recent paper [2] of Carlson, Lin, Nakano and Parshall, concerning elements in a restricted Lie algebra in characteristic $p$ which satisfy $x^{[p]}=0$.

The organization of this paper is as follows. In section 1 we give the values $d_{\Phi, r}$ and prove some results about them. Next in section 2 we consider unipotent elements, and calculate the minimal dimensions of centralizers of unipotent elements having a prescribed power equal to the identity. Finally we apply these results in section 3 to consider arbitrary elements, and prove the various statements involved in Theorem 1 .

\section{The values $d_{\Phi, r}$}

We begin with some notation. Given $x \in \mathbb{Z}$, we set $\epsilon_{x}=1$ or 0 according to whether $x$ is odd or even. For $y \in \mathbb{R}$ we write $\lceil y\rceil$ for the least integer greater than or equal to $y$.

Let $\Phi$ be a simple root system of rank $\ell$, and let $h=\frac{|\Phi|}{\ell}$ be the Coxeter number of $\Phi$; take $r \in \mathbb{N}$. For $\Phi$ of classical type, write

$$
h=z r+e \quad \text { with } z \in \mathbb{Z}, 0 \leq e<r,
$$

and set

$$
\begin{aligned}
& d_{A_{\ell}, r}=z^{2} r+e(2 z+1)-1, \\
& d_{B_{\ell}, r}=\frac{1}{2}\left(z^{2} r+e(2 z+1)\right)+\epsilon_{r}\left\lceil\frac{z}{2}\right\rceil, \\
& d_{C_{\ell}, r}=\frac{1}{2}\left(z^{2} r+e(2 z+1)\right)+\epsilon_{r}\left\lceil\frac{z}{2}\right\rceil, \\
& d_{D_{\ell}, r}=\frac{1}{2}\left(z^{2} r+e(2 z+1)\right)+\epsilon_{r}\left\lceil\frac{z}{2}\right\rceil+z+1-\epsilon_{z} .
\end{aligned}
$$

For $\Phi$ of exceptional type, write

$$
\epsilon_{\Phi, r}= \begin{cases}1 & \text { if }(\Phi, r)=\left(F_{4}, 5\right),\left(E_{6}, 2\right),\left(E_{6}, 4\right),\left(E_{8}, 11\right), \\ 0 & \text { otherwise }\end{cases}
$$

then set

$$
d_{\Phi, r}=\ell+2 \max \left(0,\left\lceil\frac{1}{2}\left(|\Phi| / r-\ell\left(1-\delta_{1 r}\right)\right)\right\rceil+\epsilon_{\Phi, r}\right),
$$

where $\delta_{* *}$ is the Kronecker delta. For convenience we list the values of $d_{\Phi, r}$ for $r<h$ and $\Phi$ exceptional in the following table:

\begin{tabular}{r|r|r|r|r|r||r|r|r|r|r||r|r|r||r|r||c|c}
$r$ & \multicolumn{1}{|c|}{$G_{2}$} & $F_{4}$ & $E_{6}$ & \multicolumn{1}{|c|}{$E_{7}$} & \multicolumn{1}{|c|}{$E_{8}$} & $r$ & $F_{4}$ & $E_{6}$ & $E_{7}$ & $E_{8}$ & $r$ & $E_{7}$ & $E_{8}$ & $r$ & $E_{8}$ & $r$ & $E_{8}$ \\
\hline & & & & & & 6 & 8 & 12 & 21 & 40 & 12 & 11 & 20 & 18 & 14 & 24 & 10 \\
1 & 14 & 52 & 78 & 133 & 248 & 7 & 8 & 12 & 19 & 36 & 13 & 11 & 20 & 19 & 14 & 25 & 10 \\
2 & 6 & 24 & 38 & 63 & 120 & 8 & 6 & 10 & 17 & 30 & 14 & 9 & 18 & 20 & 12 & 26 & 10 \\
3 & 4 & 16 & 24 & 43 & 80 & 9 & 6 & 8 & 15 & 28 & 15 & 9 & 16 & 21 & 12 & 27 & 10 \\
4 & 4 & 12 & 20 & 33 & 60 & 10 & 6 & 8 & 13 & 24 & 16 & 9 & 16 & 22 & 12 & 28 & 10 \\
5 & 4 & 12 & 16 & 27 & 48 & 11 & 6 & 8 & 13 & 24 & 17 & 9 & 16 & 23 & 12 & 29 & 10
\end{tabular}

Our first result shows why it suffices to list $d_{\Phi, r}$ for $r<h$.

Lemma 1.1. If $r \geq h$, then $d_{\Phi, r}=\ell$.

Proof. First consider $\Phi$ of classical type. If $r=h$, then $z=1$ and $e=0$, so that $z^{2} r+e(2 z+1)=h$; since $h=\ell+1,2 \ell, 2 \ell, 2 \ell-2$ for $\Phi=A_{\ell}, B_{\ell}, C_{\ell}, D_{\ell}$ respectively, in each case we obtain $d_{\Phi, r}=\ell$. If instead $r>h$, then $z=0$ and $e=h$, and the result again follows. For $\Phi$ of exceptional type, if $r \geq h$ we have $\frac{|\Phi|}{r}-\ell \leq 0$ (and $\left.\delta_{1 r}=\epsilon_{\Phi, r}=0\right)$, and the result is immediate. 
Our next result establishes lower bounds on the values $d_{\Phi, r}$. Recall that a prime $p_{1}$ is said to be very good for $\Phi$ if either $\Phi=A_{\ell}$ and $p_{1}$ does not divide $\ell+1$, or $\Phi$ is not of type $A$ and $p_{1}$ is good for $\Phi$ (so that $p_{1} \neq 2$ if $\Phi=B_{\ell}, C_{\ell}, D_{\ell}, p_{1} \neq 2,3$ if $\Phi=G_{2}, F_{4}, E_{6}, E_{7}$, and $p_{1} \neq 2,3,5$ if $\left.\Phi=E_{8}\right)$.

Lemma 1.2. We have $d_{\Phi, r} \geq \frac{|\Phi|}{r}$; moreover if $r$ is a product of very good primes, then $d_{\Phi, r} \geq \frac{|\Phi|+\ell}{r}$.

Proof. Begin with the first inequality. For $\Phi$ of exceptional type, this is clear from the definition of $d_{\Phi, r}$. For $\Phi$ of classical type we find that we have

$$
r d_{\Phi, r}-|\Phi|= \begin{cases}z r+(e-1)(r-e) & \text { if } \Phi=A_{\ell}, \\ \frac{1}{2} e(r-e)+r \epsilon_{r}\left\lceil\frac{z}{2}\right\rceil & \text { if } \Phi=B_{\ell} \text { or } C_{\ell}, \\ \frac{1}{2}(e+2)(r-e)+r\left(\epsilon_{r}\left\lceil\frac{z}{2}\right\rceil-\epsilon_{z}\right) & \text { if } \Phi=D_{\ell} ;\end{cases}
$$

in each case the expression given is non-negative, as required (note that if $r$ is even the last may be written as $\frac{1}{2} e(r-e-2)+r\left(1-\epsilon_{z}\right)$, and we have $e \leq r-2$ because the equation $2 \ell-2=h=z r+e$ forces $e$ to be even).

Now assume that $r$ is a product of very good primes. For $\Phi$ of exceptional type the second inequality is clear by inspection of the above table. For $\Phi$ of classical type we subtract $\ell$ from the expressions in the previous paragraph to obtain

$$
r d_{\Phi, r}-|\Phi|-\ell= \begin{cases}(e-1)(r-e-1) & \text { if } \Phi=A_{\ell}, \\ \frac{1}{2} e(r-e-1)+r\left(\epsilon_{r}\left\lceil\frac{z}{2}\right\rceil-\frac{z}{2}\right) & \text { if } \Phi=B_{\ell} \text { or } C_{\ell}, \\ \frac{1}{2}(e+2)(r-e-1)+r\left(\epsilon_{r}\left\lceil\frac{z}{2}\right\rceil-\frac{z}{2}-\epsilon_{z}\right) & \text { if } \Phi=D_{\ell} .\end{cases}
$$

For $\Phi=A_{\ell}$ the condition that $r$ is a product of very good primes implies that $e>0$, so that the expression above is non-negative. For the other classical types $r$ must be odd and so $\epsilon_{r}=1$; this makes the expressions for $B_{\ell}$ and $C_{\ell}$ non-negative, while for $D_{\ell}$ we need only consider the case where $z$ is odd. Here the equation $2 \ell-2=h=z r+e$ forces $e$ to be odd as well, so that $e \leq r-2$, and then we have

$$
r d_{\Phi, r}-|\Phi|-\ell=\frac{1}{2}(e+2)(r-e-1)-\frac{1}{2} r=\frac{1}{2}(e+1)(r-e-2) \geq 0
$$

as required.

We shall also require the following lemma.

Lemma 1.3. With the notation established, if $m>1$, then $d_{\Phi, r} \geq d_{\Phi, m r}$, with equality if and only if $d_{\Phi, r}=\ell$.

Proof. For $\Phi$ of exceptional type this is immediate by inspection of the table above. For $\Phi$ of classical type we write $z=z_{1} m+t$ with $0 \leq t<m$; then $h=z_{1}(m r)+$ $(t r+e)$ and $0 \leq t r+e<m r$. We then calculate

$$
\begin{aligned}
d_{A_{\ell}, r}-d_{A_{\ell}, m r} & =z^{2} r+e(2 z+1)-1-z_{1}^{2} m r-(t r+e)\left(2 z_{1}+1\right)+1 \\
& =z_{1}(m-1)\left(z_{1} m r+2 t r+2 e\right)+\operatorname{tr}(t-1)+2 t e \\
& \geq 0,
\end{aligned}
$$


with equality requiring $z_{1}=0$, which forces $m r>h$ and $d_{A_{\ell}, r}=d_{A_{\ell}, m r}=\ell$. Similarly

$$
\begin{aligned}
d_{B_{\ell}, r}-d_{B_{\ell}, m r}= & \frac{1}{2} z^{2} r+\frac{1}{2} e(2 z+1)+\epsilon_{r}\left\lceil\frac{z}{2}\right\rceil \\
& -\frac{1}{2} z_{1}^{2} m r-\frac{1}{2}(t r+e)\left(2 z_{1}+1\right)-\epsilon_{m r}\left\lceil\frac{z_{1}}{2}\right\rceil \\
= & \frac{1}{2}\left(z_{1}(m-1)\left(z_{1} m r+2 t r+2 e\right)+\operatorname{tr}(t-1)+2 t e\right) \\
& +\left(\epsilon_{r}\left\lceil\frac{z_{1} m+t}{2}\right\rceil-\epsilon_{m r}\left\lceil\frac{z_{1}}{2}\right\rceil\right) \\
\geq & 0,
\end{aligned}
$$

with equality again requiring $z_{1}=0$ and so $d_{B_{\ell}, r}=d_{B_{\ell}, m r}=\ell$; the calculation for $d_{C_{\ell}, r}$ is identical. Finally we have

$$
\begin{aligned}
d_{D_{\ell}, r}-d_{D_{\ell}, m r}= & \frac{1}{2} z^{2} r+\frac{1}{2} e(2 z+1)+\epsilon_{r}\left\lceil\frac{z}{2}\right\rceil+z+1-\epsilon_{z} \\
& -\frac{1}{2} z_{1}^{2} m r-\frac{1}{2}(t r+e)\left(2 z_{1}+1\right)-\epsilon_{m r}\left\lceil\frac{z_{1}}{2}\right\rceil-z_{1}-1+\epsilon_{z_{1}} \\
= & \frac{1}{2}\left(z_{1}(m-1)\left(z_{1} m r+2 t r+2 e\right)+\operatorname{tr}(t-1)+2 t e\right) \\
& +\left(\epsilon_{r}\left\lceil\frac{z_{1} m+t}{2}\right\rceil-\epsilon_{m r}\left\lceil\frac{z_{1}}{2}\right\rceil\right)+z_{1}(m-1)+\left(t+\epsilon_{z_{1}}-\epsilon_{z_{1} m+t}\right) \\
\geq & 0,
\end{aligned}
$$

with equality once more requiring $z_{1}=0$ and so $d_{D_{\ell}, r}=d_{D_{\ell}, m r}=\ell$.

\section{UNIPOTENT ELEMENTS}

In this section we shall establish the minimal centralizer dimension of unipotent elements of prescribed order in a simple algebraic group $H$. These results will be utilized in the following section to prove the main theorem of this paper.

We shall proceed by considering the Jordan structure of unipotent elements. For $H$ classical we take the action of $H$ on its natural module, and use results linking Jordan structure and centralizer structure due to [20] for good characteristic and 7] for types $B, C$ and $D$ in characteristic 2 . For $H$ exceptional we take the adjoint action of $H$, and use results of [8] on Jordan structure and [3, 5, 14, 15, 17, 18, on centralizer structure. (Note that [15] contains some errors involving centralizers in $E_{8}$ in characteristic 2, but these concern component groups, not the structure of connected centralizers.)

We use the following notation throughout this section and the next. Let $q$ be a power of the characteristic $p$ of the simple algebraic group $H$, and set

$$
d_{q}(H)=\min _{u \in H_{[q]}} \operatorname{dim} C_{H}(u) .
$$

Given $x \in \mathbb{Z}$ we set $\zeta_{x}=1$ or 0 according to whether $x=0$ or $x \neq 0$, and as above set $\epsilon_{x}=1$ or 0 according to whether $x$ is odd or even. For $y \in \mathbb{R}$ we write $\lfloor y\rfloor$ for the greatest integer less than or equal to $y$, and $\lceil y\rceil$ for the least integer greater than or equal to $y$.

Lemma 2.1. Take $m \in \mathbb{N}$.

(i) If $m+1=\gamma q+\delta$ with $0 \leq \delta<q$, then $d_{q}\left(A_{m}\right)=\gamma^{2} q+(2 \gamma+1) \delta-1$.

(ii) If $m=\gamma q+\delta$ with $0 \leq \delta<q$, then $d_{q}\left(A_{m}\right)=\gamma^{2} q+(2 \gamma+1)(\delta+1)-1$.

Proof. Take $H$ of type $A_{m}$; take $u \in H_{[q]}$ and write $r_{i}$ for the number of Jordan blocks of size $i$ in the action of $u$ on the natural module, so that we have $\sum i r_{i}=$ 
$m+1$, and $r_{i}=0$ for $i>q$ since $u^{q}=1$. By [20],

$$
\operatorname{dim} C_{H}(u)=\sum_{i}\left(r_{i}+r_{i+1}+\cdots+r_{q}\right)^{2}-1 ;
$$

among elements of $H_{[q]}$ this is clearly minimized when at most one Jordan block has size less than $q$.

In (i) we require Jordan structure $q^{\gamma}, \delta$, so that $r_{q}=\gamma, r_{\delta}=1$ if $\delta>0$ and $r_{i}=0$ otherwise. This gives $\operatorname{dim} C_{H}(u)=\delta(\gamma+1)^{2}+(q-\delta) \gamma^{2}-1=\gamma^{2} q+(2 \gamma+1) \delta-1$ as required. Now consider (ii). If $\delta<q-1$, then we have Jordan structure $q^{\gamma}, \delta+1$, whence $\operatorname{dim} C_{H}(u)=(\delta+1)(\gamma+1)^{2}+(q-\delta-1) \gamma^{2}-1=\gamma^{2} q+(2 \gamma+1)(\delta+1)-1$ as required; if $\delta=q-1$ the Jordan structure is $q^{\gamma+1}$, giving $\operatorname{dim} C_{H}(u)=q(\gamma+1)^{2}-1$, again as required.

Lemma 2.2. Take $m \in \mathbb{N}$.

(i) If $2 m=\gamma q+\delta$ with $0 \leq \delta<q$, then

$$
d_{q}\left(C_{m}\right)=\frac{1}{2} \gamma^{2} q+\frac{1}{2}(2 \gamma+1) \delta+\epsilon_{q}\left\lceil\frac{\gamma}{2}\right\rceil .
$$

(ii) If $2 m+1=\gamma q+\delta$ with $0 \leq \delta<q$, then

$$
d_{q}\left(C_{m+1}\right)=\frac{1}{2} \gamma^{2} q+\frac{1}{2}(2 \gamma+1)(\delta+1)+\epsilon_{q}\left\lceil\frac{\gamma}{2}\right\rceil .
$$

(iii) If $2 m+1=\gamma q+\delta$ with $0 \leq \delta<q$, then

$$
d_{q}\left(C_{m}\right)=\frac{1}{2} \gamma^{2} q+\frac{1}{2}(2 \gamma+1)(\delta-1)+\epsilon_{q}\left\lceil\frac{\gamma}{2}\right\rceil .
$$

Proof. Take $H$ of type $C_{k}$; as in the previous result take $u \in H_{[q]}$ and write $r_{i}$ for the number of Jordan blocks of size $i$ in the action of $u$ on the natural module, so that $\sum i r_{i}=2 k$ and $r_{i}=0$ for $i>q$. We must have $r_{i}$ even for all odd $i$. If $p$ is odd, [20] gives $\operatorname{dim} C_{H}(u)=\frac{1}{2} \sum_{i}\left(r_{i}+r_{i+1}+\cdots+r_{q}\right)^{2}+\frac{1}{2} \sum_{i \text { odd }} r_{i}$; if $p=2$, then $\operatorname{dim} C_{H}(u)$ is not determined simply by the Jordan structure, but [7] gives a formula whose minimal value reduces to that for odd characteristic. As in the previous lemma, the optimal Jordan structure will involve as many blocks of size $q$ as possible.

Begin with (i), so that $k=m$. If $p=2$, then either $q=1$, in which case $u=1$ and $\operatorname{dim} C_{H}(u)=\operatorname{dim} H=2 m^{2}+m$; or $q>1$, when we have Jordan structure $q^{\gamma}, \delta$ (note that $\delta=2 m-\gamma q$ is even here), giving $\operatorname{dim} C_{H}(u)=\frac{1}{2}\left[\delta(\gamma+1)^{2}+(q-\delta) \gamma^{2}\right]=$ $\frac{1}{2} \gamma^{2} q+\frac{1}{2}(2 \gamma+1) \delta$. If instead $p$ is odd, we take separately the cases where $\gamma$ (and hence $\delta$ ) is even and odd: if $\gamma$ is even the Jordan structure is $q^{\gamma}, \delta$, whence $\operatorname{dim} C_{H}(u)=\frac{1}{2}\left[\delta(\gamma+1)^{2}+(q-\delta) \gamma^{2}+\gamma\right]=\frac{1}{2} \gamma^{2} q+\frac{1}{2}(2 \gamma+1) \delta+\frac{1}{2} \gamma$; if $\gamma$ is odd the Jordan structure is $q^{\gamma-1}, q-1, \delta+1$ (or $q^{\gamma-1},(q-1)^{2}$ if $\delta=q-2$ ), whence $\operatorname{dim} C_{H}(u)=\frac{1}{2}\left[(\delta+1)(\gamma+1)^{2}+(q-\delta-2) \gamma^{2}+(\gamma-1)^{2}+\gamma-1\right]=$ $\frac{1}{2} \gamma^{2} q+\frac{1}{2}(2 \gamma+1) \delta+\frac{1}{2}(\gamma+1)$. Thus in all cases the minimal centralizer dimension is $\frac{1}{2} \gamma^{2} q+\frac{1}{2}(2 \gamma+1) \delta+\epsilon_{q}\left\lceil\frac{\gamma}{2}\right\rceil$, as required.

For (ii) and (iii) we proceed by comparing with (i). For (ii) we have $k=m+1$; thus if we write $2 k=\gamma^{\prime} q+\delta^{\prime}$ with $0 \leq \delta^{\prime}<q$, we have $\gamma^{\prime} q+\delta^{\prime}-1=\gamma q+\delta$. If $\delta<q-1$ we then have $\gamma^{\prime}=\gamma, \delta^{\prime}=\delta+1$ and the minimal centralizer dimension is $\frac{1}{2}{\gamma^{\prime}}^{2} q+\frac{1}{2}\left(2 \gamma^{\prime}+1\right) \delta^{\prime}+\epsilon_{q}\left\lceil\frac{\gamma^{\prime}}{2}\right\rceil=\frac{1}{2} \gamma^{2} q+\frac{1}{2}(2 \gamma+1)(\delta+1)+\epsilon_{q}\left\lceil\frac{\gamma}{2}\right\rceil$; if $\delta=q-1$ we have $\gamma^{\prime}=\gamma+1, \delta^{\prime}=0$ and the minimal centralizer dimension is $\frac{1}{2}(\gamma+1)^{2} q+\epsilon_{q}\left\lceil\frac{\gamma+1}{2}\right\rceil=$ $\frac{1}{2} \gamma^{2} q+\frac{1}{2}(2 \gamma+1) q+\epsilon_{q}\left\lceil\frac{\gamma}{2}\right\rceil$ (note that if $q$ is odd here, then $\gamma^{\prime}=\gamma+1$ must be even, in which case $\left.\left\lceil\frac{\gamma+1}{2}\right\rceil=\left\lceil\frac{\gamma}{2}\right\rceil\right)$. In either case we obtain the required formula. For (iii) we have $k=m$; thus if we set $2 k=\gamma^{\prime} q+\delta^{\prime}$ with $0 \leq \delta^{\prime}<q$ we have 
$\gamma^{\prime} q+\delta^{\prime}+1=\gamma q+\delta$. If $\delta>0$, then $\gamma^{\prime}=\gamma, \delta^{\prime}=\delta-1$ and the minimal centralizer dimension is $\frac{1}{2} \gamma^{2} q+\frac{1}{2}(2 \gamma+1)(\delta-1)+\epsilon_{q}\left\lceil\frac{\gamma}{2}\right\rceil$; if $\delta=0$, then $\gamma^{\prime}=\gamma-1, \delta^{\prime}=q-1$ and the minimal centralizer dimension is $\frac{1}{2}(\gamma-1)^{2} q+\frac{1}{2}(2 \gamma-1)(q-1)+\epsilon_{q}\left\lceil\frac{\gamma-1}{2}\right\rceil=$ $\frac{1}{2}\left(\gamma^{2} q-2 \gamma+1\right)+\epsilon_{q}\left\lceil\frac{\gamma-1}{2}\right\rceil=\frac{1}{2} \gamma^{2} q-\frac{1}{2}(2 \gamma+1)+\epsilon_{q}\left\lceil\frac{\gamma}{2}\right\rceil$ (note that both $\gamma$ and $q$ must be odd here, so $\left.1+\epsilon_{q}\left\lceil\frac{\gamma-1}{2}\right\rceil=\epsilon_{q}\left\lceil\frac{\gamma}{2}\right\rceil\right)$. Again the required formula follows.

Lemma 2.3. Take $m \in \mathbb{N}$.

(i) If $2 m=\gamma q+\delta$ with $0 \leq \delta<q$, then

$$
d_{q}\left(B_{m}\right)=\frac{1}{2} \gamma^{2} q+\frac{1}{2}(2 \gamma+1) \delta+\epsilon_{q}\left\lceil\frac{\gamma}{2}\right\rceil .
$$

(ii) If $2 m+1=\gamma q+\delta$ with $0 \leq \delta<q$, then

$$
d_{q}\left(B_{m+1}\right)=\frac{1}{2} \gamma^{2} q+\frac{1}{2}(2 \gamma+1)(\delta+1)+\epsilon_{q}\left\lceil\frac{\gamma}{2}\right\rceil .
$$

(iii) If $2 m+1=\gamma q+\delta$ with $0 \leq \delta<q$, then

$$
d_{q}\left(B_{m}\right)=\frac{1}{2} \gamma^{2} q+\frac{1}{2}(2 \gamma+1)(\delta-1)+\epsilon_{q}\left\lceil\frac{\gamma}{2}\right\rceil
$$

Proof. Take $H$ of type $B_{k}$ and $u \in H_{[q]}$, and as before write $r_{i}$ for the number of Jordan blocks of size $i$ in the action of $u$ on the natural module, so that $\sum i r_{i}=$ $2 k+1$ and $r_{i}=0$ for $i>q$. If $p$ is odd, we must have $r_{i}$ even for all even $i$; 20] gives $\operatorname{dim} C_{H}(u)=\frac{1}{2} \sum_{i}\left(r_{i}+r_{i+1}+\cdots+r_{q}\right)^{2}-\frac{1}{2} \sum_{i \text { odd }} r_{i}$. If $p=2$, we must instead have $r_{i}$ even for all odd $i>1$; here $\operatorname{dim} C_{H}(u)$ is again not determined simply by the Jordan structure, but [7] gives a formula whose minimal value reduces to $\frac{1}{2} \sum_{i}\left(r_{i}+r_{i+1}+\cdots+r_{q}\right)^{2}-\frac{1}{2} \sum_{i \text { odd }} r_{i}-\sum_{i \text { even }} r_{i}$. Once more, the optimal Jordan structure will involve as many blocks of size $q$ as possible. Note that it suffices to prove (i), as (ii) and (iii) will then follow by identical calculations to those in the previous result.

Thus let $k=m$. If $p=2$, then either $q=1$, in which case $u=1$ and $\operatorname{dim} C_{H}(u)=$ $\operatorname{dim} H=2 m^{2}+m$; or $q>1$, when $\delta=2 m-\gamma q$ must be even and we must distinguish the cases $\delta=0$ and $\delta>0$. In the case $\delta=0$ we have Jordan structure $q^{\gamma}, 1$, giving $\operatorname{dim} C_{H}(u)=\frac{1}{2}\left[(\gamma+1)^{2}+(q-1) \gamma^{2}-1-2 \gamma\right]=\frac{1}{2} \gamma^{2} q$; for $\delta>0$ the Jordan structure is $q^{\gamma}, \delta, 1$, giving

$$
\begin{aligned}
\operatorname{dim} C_{H}(u) & =\frac{1}{2}\left[(\gamma+2)^{2}+(\delta-1)(\gamma+1)^{2}+(q-\delta) \gamma^{2}-1-2 \gamma-2\right] \\
& =\frac{1}{2} \gamma^{2} q+\frac{1}{2}(2 \gamma+1) \delta
\end{aligned}
$$

If $p$ is odd instead, as in the previous result we take the cases where $\gamma$ (and hence $\delta$ ) is even and odd separately. If $\gamma$ is even the Jordan structure is $q^{\gamma}, \delta+1$ (or $q^{\gamma+1}$ if $\delta=q-1$ ), whence

$$
\begin{aligned}
\operatorname{dim} C_{H}(u) & =\frac{1}{2}\left[(\delta+1)(\gamma+1)^{2}+(q-\delta-1) \gamma^{2}-\gamma-1\right] \\
& =\frac{1}{2} \gamma^{2} q+\frac{1}{2}(2 \gamma+1) \delta+\frac{1}{2} \gamma .
\end{aligned}
$$

If $\gamma$ is odd the Jordan structure is $q^{\gamma}, \delta, 1$ (or $q^{\gamma}, 1^{2}$ if $\delta=1$ ), whence

$$
\begin{aligned}
\operatorname{dim} C_{H}(u) & =\frac{1}{2}\left[(\gamma+2)^{2}+(\delta-1)(\gamma+1)^{2}+(q-\delta) \gamma^{2}+(\gamma-1)^{2}-\gamma-2\right] \\
& =\frac{1}{2} \gamma^{2} q+\frac{1}{2}(2 \gamma+1) \delta+\frac{1}{2}(\gamma+1)
\end{aligned}
$$

Thus in all cases the minimal centralizer dimension is $\frac{1}{2} \gamma^{2} q+\frac{1}{2}(2 \gamma+1) \delta+\epsilon_{q}\left\lceil\frac{\gamma}{2}\right\rceil$, as required. 
Lemma 2.4. Take $m \in \mathbb{N}$.

(i) If $2 m-1=\gamma q+\delta$ with $0 \leq \delta<q$, then $d_{q}\left(D_{m}\right)=\frac{1}{2} \gamma^{2} q+\frac{1}{2}(2 \gamma+1) \delta+\frac{1}{2}+$ $\epsilon_{q}\left\lceil\frac{\gamma}{2}\right\rceil-\epsilon_{\gamma}$.

(ii) If $2 m=\gamma q+\delta$ with $0 \leq \delta<q$, then $d_{q}\left(D_{m+1}\right)=\frac{1}{2} \gamma^{2} q+\frac{1}{2}(2 \gamma+1)(\delta+1)+$ $\frac{1}{2}+\epsilon_{q}\left\lceil\frac{\gamma}{2}\right\rceil-\epsilon_{\gamma}$.

(iii) If $2 m=\gamma q+\delta$ with $0 \leq \delta<q$, then $d_{q}\left(D_{m}\right)=\frac{1}{2} \gamma^{2} q+\frac{1}{2}(2 \gamma+1)(\delta-1)+$ $\frac{1}{2}+\epsilon_{q}\left\lceil\frac{\gamma}{2}\right\rceil-\epsilon_{\gamma}+2 \epsilon_{\gamma} \zeta_{\delta}$.

Proof. This is very similar to the previous result, but the details are rather more complicated. Take $H$ of type $D_{k}$ and $u \in H_{[q]}$, and again write $r_{i}$ for the number of Jordan blocks of size $i$ in the action of $u$ on the natural module, so that $\sum i r_{i}=2 k$ and $r_{i}=0$ for $i>q$. If $p$ is odd, we must again have $r_{i}$ even for all even $i$, and 20 once more gives $\operatorname{dim} C_{H}(u)=\frac{1}{2} \sum_{i}\left(r_{i}+r_{i+1}+\cdots+r_{q}\right)^{2}-\frac{1}{2} \sum_{i \text { odd }} r_{i}$. If $p=2$, we must instead have $r_{i}$ even for all odd $i$, and $\sum r_{i}$ must also be even; again, the minimal value taken by the formula in [7] reduces to

$$
\frac{1}{2} \sum_{i}\left(r_{i}+r_{i+1}+\cdots+r_{q}\right)^{2}-\frac{1}{2} \sum_{i \text { odd }} r_{i}-\sum_{i \text { even }} r_{i}
$$

First consider (i), and let $k=m$. If $p$ is odd, we separate into two cases according to the parity of $\gamma$. If $\gamma$ is odd, then $\delta$ is even and the required Jordan structure is $q^{\gamma}, \delta+1$ (or $q^{\gamma+1}$ if $\delta=q-1$ ), giving $\operatorname{dim} C_{H}(u)=\frac{1}{2} \gamma^{2} q+\frac{1}{2}(2 \gamma+1) \delta+\frac{1}{2} \gamma$; if $\gamma$ is even, then $\delta$ is odd and the Jordan structure is $q^{\gamma}, \delta, 1$ (or $q^{\gamma}, 1^{2}$ if $\delta=1$ ), and we have $\operatorname{dim} C_{H}(u)=\frac{1}{2} \gamma^{2} q+\frac{1}{2}(2 \gamma+1) \delta+\frac{1}{2}(\gamma+1)$. If $p=2$, then either $q=1$, in which case $u=1$ and $\operatorname{dim} C_{H}(u)=\operatorname{dim} H=2 m^{2}-m$; or $q>1$, when $\delta$ must be odd and we again separate into two cases according to the parity of $\gamma$. If $\gamma$ is odd, the Jordan structure is $q^{\gamma}, \delta+1$ (or $q^{\gamma+1}$ if $\delta=q-1$ ), and $\operatorname{dim} C_{H}(u)=\frac{1}{2} \gamma^{2} q+\frac{1}{2}(2 \gamma+1) \delta-\frac{1}{2}$. If $\gamma$ is even, the Jordan structure can take several forms depending on $\delta$ and $q$ : if $\delta=q-1$ we have $q^{\gamma}, q-2,2$ if $q>4$, or $4^{\gamma}, 2^{2}$ if $q=4$, or $2^{\gamma}, 1^{2}$ if $q=2$; if $3<\delta<q-1$ we have $q^{\gamma}, \delta-1,2$; if $\delta=3$ we have $q^{\gamma}, 2^{2}$; and if $\delta=1$ we have $q^{\gamma}, 1^{2}$. For each of these possibilities we find $\operatorname{dim} C_{H}(u)=\frac{1}{2} \gamma^{2} q+\frac{1}{2}(2 \gamma+1) \delta+\frac{1}{2}$. Thus in all cases the minimal centralizer dimension is $\frac{1}{2} \gamma^{2} q+\frac{1}{2}(2 \gamma+1) \delta+\frac{1}{2}+\epsilon_{q}\left\lceil\frac{\gamma}{2}\right\rceil-\epsilon_{\gamma}$, as required.

For (ii) and (iii) we again proceed by comparing with (i). For (ii) we have $k=$ $m+1$; thus if we write $2 k-1=\gamma^{\prime} q+\delta^{\prime}$ with $0 \leq \delta^{\prime}<q$, we have $\gamma^{\prime} q+\delta^{\prime}-1=\gamma q+\delta$. If $\delta<q-1$ we then have $\gamma^{\prime}=\gamma, \delta^{\prime}=\delta+1$ and the minimal centralizer dimension is $\frac{1}{2} \gamma^{\prime 2} q+\frac{1}{2}\left(2 \gamma^{\prime}+1\right) \delta^{\prime}+\frac{1}{2}+\epsilon_{q}\left\lceil\frac{\gamma^{\prime}}{2}\right\rceil-\epsilon_{\gamma^{\prime}}=\frac{1}{2} \gamma^{2} q+\frac{1}{2}(2 \gamma+1)(\delta+1)+\frac{1}{2}+\epsilon_{q}\left\lceil\frac{\gamma}{2}\right\rceil-\epsilon_{\gamma}$; if $\delta=q-1$ we have $\gamma^{\prime}=\gamma+1, \delta^{\prime}=0$ and the minimal centralizer dimension is $\frac{1}{2}(\gamma+1)^{2} q+\frac{1}{2}+\epsilon_{q}\left\lceil\frac{\gamma+1}{2}\right\rceil-\epsilon_{\gamma+1}=\frac{1}{2} \gamma^{2} q+\frac{1}{2}(2 \gamma+1) q+\frac{1}{2}+\frac{1}{2} \gamma$ (note that here $q$ is odd and $\gamma$ even). In either case we obtain the required formula. For (iii) we have $k=m$; thus if we set $2 k-1=\gamma^{\prime} q+\delta^{\prime}$ with $0 \leq \delta^{\prime}<q$ we have $\gamma^{\prime} q+\delta^{\prime}+1=\gamma q+\delta$. If $\delta>0$, then $\gamma^{\prime}=\gamma, \delta^{\prime}=\delta-1$ and the minimal centralizer dimension is $\frac{1}{2} \gamma^{2} q+\frac{1}{2}(2 \gamma+1)(\delta-1)+\frac{1}{2}+\epsilon_{q}\left\lceil\frac{\gamma}{2}\right\rceil-\epsilon_{\gamma}$. If on the other hand $\delta=0$, then $\gamma^{\prime}=\gamma-1, \delta^{\prime}=q-1$ and the minimal centralizer dimension is $\frac{1}{2}(\gamma-1)^{2} q+\frac{1}{2}(2 \gamma-1)(q-1)+\frac{1}{2}+\epsilon_{q}\left\lceil\frac{\gamma-1}{2}\right\rceil-\epsilon_{\gamma-1}=\frac{1}{2} \gamma^{2} q-\frac{1}{2}(2 \gamma+1)+1 \frac{1}{2}+\epsilon_{q}\left\lceil\frac{\gamma-1}{2}\right\rceil-$ $\epsilon_{\gamma-1}=\frac{1}{2} \gamma^{2} q-\frac{1}{2}(2 \gamma+1)+\frac{1}{2}+\epsilon_{q}\left\lceil\frac{\gamma}{2}\right\rceil+\epsilon_{\gamma}$ (note that $\gamma q$ must be even here, so that if $q$ is odd, then $\gamma$ must be even, whence $\epsilon_{q}\left\lceil\frac{\gamma-1}{2}\right\rceil=\epsilon_{q}\left\lceil\frac{\gamma}{2}\right\rceil$; and $1-\epsilon_{\gamma-1}=\epsilon_{\gamma}$ ). Again the required formula follows. 
We may summarize the above results as follows.

Lemma 2.5. If $a=c q+d$ with $0 \leq d<q$ and $\zeta \in\{0,1\}$ we have the following:

(i) $d_{q}\left(A_{a-\zeta}\right)=c^{2} q+(2 c+1)(a-\zeta-c q+1)-1$;

(ii) $d_{q}\left(B_{\left\lceil\frac{a}{2}\right\rceil-\epsilon_{a} \zeta}\right)=\frac{1}{2} c^{2} q+(2 c+1)\left(\left\lceil\frac{a}{2}\right\rceil-\epsilon_{a} \zeta-\frac{1}{2} c q\right)+\epsilon_{q}\left\lceil\frac{c}{2}\right\rceil$;

(iii) $d_{q}\left(C_{\left\lceil\frac{a}{2}\right\rceil-\epsilon_{a} \zeta}\right)=\frac{1}{2} c^{2} q+(2 c+1)\left(\left\lceil\frac{a}{2}\right\rceil-\epsilon_{a} \zeta-\frac{1}{2} c q\right)+\epsilon_{q}\left\lceil\frac{c}{2}\right\rceil$;

(iv) $d_{q}\left(D_{\left\lceil\frac{a+1}{2}\right\rceil-\epsilon_{a+1} \zeta}\right)=\frac{1}{2} c^{2} q+(2 c+1)\left(\left\lceil\frac{a+1}{2}\right\rceil-\epsilon_{a+1} \zeta-\frac{1}{2} c q\right)+\epsilon_{q}\left\lceil\frac{c}{2}\right\rceil-c-\epsilon_{c}+$ $2 \epsilon_{c(a+1)} \zeta_{a-c q} \zeta$.

We have also shown the following.

Corollary 2.6. Take $m \in \mathbb{N}$.

(i) If $m+1=\gamma q+\delta$ with $0 \leq \delta<q$, then $d_{q}\left(A_{m+1}\right)-d_{q}\left(A_{m}\right)=2 \gamma+1$.

(ii) If $2 m+1=\gamma q+\delta$ with $0 \leq \delta<q$, then $d_{q}\left(B_{m+1}\right)-d_{q}\left(B_{m}\right)=2 \gamma+1$.

(iii) If $2 m+1=\gamma q+\delta$ with $0 \leq \delta<q$, then $d_{q}\left(C_{m+1}\right)-d_{q}\left(C_{m}\right)=2 \gamma+1$.

(iv) If $2 m=\gamma q+\delta$ with $0 \leq \delta<q$, then $d_{q}\left(D_{m+1}\right)-d_{q}\left(D_{m}\right)=2 \gamma+1-2 \epsilon_{\gamma} \zeta_{\delta}$.

We now turn to the exceptional groups; here we simply obtain the values of $d_{q}(H)$ by comparing the Jordan structure of unipotent elements on the adjoint module given in 8 , with the centralizer structure as given in [3, 5, 14, 15, 17, 18. We find the following.

Lemma 2.7. If $H$ is of exceptional type with root system $\Psi$, then $d_{q}(H)=d_{\Psi, q}$.

\section{The General CASE}

In this section we shall consider arbitrary elements of a simple algebraic group $G$ defined over an algebraically closed field of characteristic $p$, and shall prove the various statements involved in Theorem 1 We begin with some notation which will be used throughout this section. Let $\Phi$ be the root system of $G$, taken with respect to some maximal torus $T$, and let $\Pi=\left\{\alpha_{1}, \ldots, \alpha_{\ell}\right\}$ be a fundamental system, numbered in accordance with [1. Planches I-IX]; write $\alpha_{0}$ for the highest root of $\Phi$ with respect to $\Pi$, set $m_{0}=1$ and define $m_{i}$ for $1 \leq i \leq \ell$ by

$$
\alpha_{0}=\sum_{i=1}^{\ell} m_{i} \alpha_{i}
$$

Let $h=\sum_{i=0}^{\ell} m_{i}$ be the Coxeter number of $\Phi$. Now take $r \in \mathbb{N}$, and let the $p^{\prime}$-part and $p$-part of $r$ be $n$ and $q$, respectively, so that $r=n q$. Given $g \in G_{[r]}$, let the Jordan decomposition of $g$ be $g=s u=u s$, where $s$ is semisimple and $u$ unipotent; we then have $s \in G_{[n]}$ and $u \in\left(C_{G}(s)^{0}\right)_{[q]}$. Extending the notation of the previous section, we write

$$
d_{r}(G)=\min _{g \in G_{[r]}} \operatorname{dim} C_{G}(g)
$$

we then have

$$
d_{r}(G)=\min _{s \in G_{[n]}} d_{q}\left(C_{G}(s)^{0}\right)=\min _{Z=C_{G}(s)^{0}, s \in G_{[n]}} d_{q}(Z),
$$

where we set $d_{q}\left(H_{1} \ldots H_{t} T^{\prime}\right)=d_{q}\left(H_{1}\right)+\cdots+d_{q}\left(H_{t}\right)$ for $H_{1}, \ldots, H_{t}$ simple and $T^{\prime}$ a torus. We can immediately provide an important interpretation of the number $d_{r}(G)$.

Lemma 3.1. With the notation established, $\operatorname{codim} G_{[r]}=d_{r}(G)$. 
Proof. Taking $g=s u$ as above, by conjugation we may assume $s \in T$; as $\left|T_{[n]}\right|=n^{\ell}$, and $C_{G}(s)^{0}$ contains finitely many unipotent classes, it follows that the number of classes in $G_{[r]}$ is finite. Thus

$$
\operatorname{dim} G_{[r]}=\max _{g \in G_{[r]}} \operatorname{dim} g^{G},
$$

and hence

$$
\operatorname{codim} G_{[r]}=\operatorname{dim} G-\max _{g \in G_{[r]}} \operatorname{dim} g^{G}=\min _{g \in G_{[r]}} \operatorname{dim} C_{G}(g)=d_{r}(G),
$$

as required.

Our approach to calculating the value of $d_{r}(G)$ will be to use a result of Hartley and Kuzucuoğlu in [6, given $n$, to restrict the possibilities for the connected centralizer $Z$ of a semisimple element of order $n$. Among the possible groups $Z$ we then select one having minimal value of $d_{q}(Z)$, using the results of the previous section. We shall observe that this minimal value in fact depends only on $r$ and not on the factorization $r=n q$, so that it is independent of the characteristic $p$.

Unless otherwise stated, we assume from now on that

$$
G \text { is of adjoint type. }
$$

At the end of this section we shall consider the case of arbitrary isogeny type.

The result from [6] which we need is (part of) Theorem 4.2 there, and follows the approach of Deriziotis in 4]; under our assumption on the isogeny type of $G$ it may be stated as follows. Choose non-negative integers $b_{0}, b_{1}, \ldots, b_{\ell}$ with $\operatorname{gcd}\left(b_{0}, b_{1}, \ldots, b_{\ell}\right)=1$ satisfying $\sum_{i=0}^{\ell} b_{i} m_{i}=n$. The roots $\alpha_{i}$ (or $-\alpha_{0}$ in the case $i=0$ ) for which $b_{i}=0$ then form a simple system $\Psi$ whose corresponding subsystem subgroup of $G$ is the centralizer of a semisimple element of order $n$; moreover, up to conjugacy all centralizers of semisimple elements of order $n$ arise in this way.

Now from this result it follows that if $n \geq h$, then by choosing $b_{1}=\cdots=b_{\ell}=1$ and $b_{0}=n+1-h$ we may take $Z=T$, whence $d_{r}(G)=\ell=d_{\Phi, r}$ as required (note that if $n \geq h$, then certainly $r \geq h$ ); it therefore suffices to assume in what follows that

$$
n<h \text {. }
$$

The following result shows that in this case we may substantially restrict the nonnegative integers $b_{i}$ which need be considered.

Lemma 3.2. With the notation established, if $n<h$ the minimal value of $d_{q}(Z)$ occurs for some $b_{0}, b_{1}, \ldots, b_{\ell}$ with $b_{i} \in\{0,1\}$ for all $i$.

Proof. Take $b_{0}, b_{1}, \ldots, b_{\ell}$ satisfying $\operatorname{gcd}\left(b_{0}, b_{1}, \ldots, b_{\ell}\right)=1$ and $\sum_{i=0}^{\ell} b_{i} m_{i}=n$. Since $n<h=\sum_{i=0}^{\ell} m_{i}$ we must have $b_{i}=0$ for some $i$; let $v=\min \left\{m_{i}: b_{i}=0\right\}$. We shall show that if not all the $b_{i}$ are 0 or 1 , then it is always possible to replace some of them in such a way that the conditions are still satisfied and the value of $d_{q}(Z)$ is either reduced or unchanged, but the quantity $\Delta=h^{2}|\Psi|+h \sum_{i=0}^{\ell} b_{i}+v$ is decreased; thus after a finite number of steps this process must terminate in a sequence $b_{0}, b_{1}, \ldots, b_{\ell}$ in which all terms are 0 or 1 as required.

Thus assume $b_{x}>1$ for some $x$; choose $y$ such that $m_{y}=v$ and $b_{y}=0$. We split into two cases according to the relative sizes of $m_{x}$ and $m_{y}$. First assume $m_{x} \geq m_{y}$. If $m_{x}=1$ we may replace the pair $\left(b_{x}, b_{y}\right)$ by $\left(b_{x}-1,1\right)$; if $m_{x}>1$ and $y=0$ replace $\left(b_{x}, b_{y}\right)$ by $\left(1,\left(b_{x}-1\right) m_{x}\right)$; if $m_{x}>1$ and $y \neq 0$ replace the triple 
$\left(b_{x}, b_{y}, b_{0}\right)$ by $\left(b_{x}-1,1, b_{0}+m_{x}-m_{y}\right)$. In each instance we obtain $b_{0}{ }^{\prime}, b_{1}{ }^{\prime}, \ldots, b_{\ell}{ }^{\prime}$ satisfying the required conditions with $\Psi^{\prime}=\Psi \backslash\left\{\alpha_{y}\right\}$, whence $d_{q}\left(Z^{\prime}\right) \leq d_{q}(Z)$; as $\left|\Psi^{\prime}\right|=|\Psi|-1$ and $h \sum_{i=0}^{\ell} b_{i}{ }^{\prime}+v^{\prime} \leq h n+v^{\prime}<h(h-1)+h=h^{2}$ we clearly have $\Delta^{\prime}<\Delta$ as required.

Now assume that $m_{x}<m_{y}$. Here we use an observation relating to the extended Dynkin diagram; we recall that this has nodes $0,1, \ldots, \ell$ corresponding to roots $-\alpha_{0}, \alpha_{1}, \ldots, \alpha_{\ell}$. Set $m=\max \left\{m_{i}: 0 \leq i \leq \ell\right\}$; then there is a sequence $0=$ $k_{1}, k_{2}, \ldots, k_{m}$ of nodes satisfying $m_{k_{t}}=t$ for $t \leq m$ and with $k_{t}$ joined to $k_{t^{\prime}}$ if and only if $\left|t-t^{\prime}\right|=1$. Now the choice of $y$ implies that for any node $t$ with $m_{t}<m_{y}$ we must have $b_{t}>0$; thus by interchanging the values $b_{x}$ and $b_{k_{m_{x}}}$ if necessary we may assume that $k_{m_{x}}=x$, i.e., the node $x$ occurs in the sequence. Let $k_{m_{y}}=z^{\prime}$ and $k_{m_{y}-1}=z$, while if $x \neq 0$ let $k_{m_{x}-1}=x^{\prime}$; then $b_{z}>0$, again by the choice of $y$, and if $b_{z^{\prime}}=0$ we may assume that $y$ is chosen to be $z^{\prime}$. We therefore have $1=m_{0} \leq m_{x} \leq m_{z}<m_{z}+1=m_{y}$ with $0, x$ and $z$ all appearing in the sequence, and if $x \neq 0$, then $x^{\prime}$ is also in the sequence with $m_{x^{\prime}}=m_{x}-1$. Now if $m_{0}=m_{x}=m_{z}$ we may replace the pair $\left(b_{x}, b_{y}\right)$ by $\left(b_{x}-2,1\right)$; if $m_{0}<m_{x}=m_{z}$ replace the triple $\left(b_{0}, b_{x}, b_{y}\right)$ by $\left(b_{0}-1, b_{x}-1,1\right)$; if $m_{0}=m_{x}<m_{z}$ replace $\left(b_{x}, b_{z}, b_{y}\right)$ by $\left(b_{x}-1, b_{z}-1,1\right)$; lastly if $m_{0}<m_{x}<m_{z}$ replace the quadruple $\left(b_{x^{\prime}}, b_{x}, b_{z}, b_{y}\right)$ by $\left(b_{x^{\prime}}+1, b_{x}-1, b_{z}-1,1\right)$. In every instance $\left|\Psi^{\prime}\right| \leq|\Psi|$; in each but the last we have $\sum_{i=0}^{\ell} b_{i}{ }^{\prime}<\sum_{i=0}^{\ell} b_{i}$, while in the last $\sum_{i=0}^{\ell} b_{i}{ }^{\prime}=\sum_{i=0}^{\ell} b_{i}$ but if $\left|\Psi^{\prime}\right|=|\Psi|$, then $v^{\prime}=m_{z}=v-1$. Thus the value of $\Delta$ decreases as required; and one irreducible component of $\Psi$ (that containing $\alpha_{y}$ ) is reduced in rank by 1 , while at most an $A_{1}$ component is introduced (containing $-\alpha_{0}$ in the first two instances and $\alpha_{z}$ in the second two), so clearly $d_{q}\left(Z^{\prime}\right) \leq d_{q}(Z)$. This completes the proof.

We may now work our way through the types of irreducible root system.

Proposition 3.3. With the notation established, $d_{r}\left(A_{\ell}\right)=d_{A_{\ell}, r}$.

Proof. We have $h=\ell+1$; and $m_{i}=1$ for all $0 \leq i \leq \ell$. By Lemma 3.2 we therefore require coefficients $b_{0}, b_{1}, \ldots, b_{\ell}$ with $n$ of the $b_{i}$ equal to 1 and the remainder equal to 0 . Suppose $b_{i_{1}}=\cdots=b_{i_{n}}=1$ with $i_{1}<\cdots<i_{n}$; we may assume $i_{1}=0$, and set $i_{n+1}=\ell+1$. We then have $Z$ as a product of $n$ factors $A_{i_{j+1}-i_{j}-1}$ (where a factor $A_{0}$ is interpreted as being trivial) and an $(n-1)$-dimensional torus $T_{n-1}$. Now if two of the factors are $A_{k}$ and $A_{k^{\prime}}$ with $k-k^{\prime}>1$, we may alter the $b_{i}$ so as to replace these two factors with $A_{k-1}$ and $A_{k^{\prime}+1}$; it follows from Corollary 2.6 (i) that $d_{q}\left(A_{k} A_{k^{\prime}}\right)-d_{q}\left(A_{k-1} A_{k^{\prime}+1}\right) \geq 0$. Thus we may assume that, for some $a$, all factors are either $A_{a}$ or $A_{a-1}$; as $\operatorname{rank} Z=\ell$ we must have

$$
Z=\left(A_{a}\right)^{b}\left(A_{a-1}\right)^{n-b} T_{n-1},
$$

where $\ell+1=a n+b$ with $0 \leq b<n$.

Now write $a=c q+d$ with $0 \leq d<q$, and apply the formulæ from Lemma 2.5)(i); we obtain

$$
\begin{aligned}
d_{q}(Z) & =b\left(c^{2} q+(2 c+1)(d+1)-1\right)+(n-b)\left(c^{2} q+(2 c+1) d-1\right)+n-1 \\
& =c^{2} n q+(2 c+1)(d n+b)-1 \\
& =c^{2} n q+(2 c+1)(\ell+1-c q n)-1 \\
& =(2 c+1)(\ell+1)-c(c+1) n q-1 \\
& =(2 c+1)(\ell+1)-c(c+1) r-1 .
\end{aligned}
$$


Since we have $z r+e=\ell+1=a n+b=(c q+d) n+b=c r+(d n+b)$ with $d n+b \leq(q-1) n+(n-1)=r-1$, it follows that $c=z$, so

$$
d_{r}\left(A_{\ell}\right)=(2 z+1)(\ell+1)-z(z+1) r-1 ;
$$

substituting from $\ell+1=z r+e$ gives $d_{r}\left(A_{\ell}\right)=d_{A_{\ell}, r}$ as required.

Proposition 3.4. With the notation established, $d_{r}\left(C_{\ell}\right)=d_{C_{\ell}, r}$.

Proof. We have $h=2 \ell$; and $m_{i}=2$ for all $1 \leq i \leq \ell-1$ while $m_{0}=m_{\ell}=1$. Here we shall need to argue separately for $n$ odd and $n$ even; first assume $n$ is odd and set $n=2 s+1$. By Lemma 3.2 we therefore require coefficients $b_{0}, b_{1}, \ldots, b_{\ell}$ in which the $b_{i}$ which are equal to 1 correspond to $s$ nodes $i$ with $1 \leq i \leq \ell-1$, together with either node 0 or node $\ell$; this means that $Z$ has $s$ factors of type $A$, one factor of type $C$ and an $s$-dimensional torus. We shall need to determine a configuration which minimizes $d_{q}(Z)$.

As in the proof of Proposition 3.3, the type $A$ factors may be assumed to take the form $\left(\tilde{A}_{x}\right)^{z}\left(\tilde{A}_{x-1}\right)^{s-z}$, for some $x$ and some $0 \leq z<s$; suppose the type $C$ factor is $C_{y}$. If we write $x=\gamma_{1} q+\delta_{1}$ and $2 y-1=\gamma_{2} q+\delta_{2}$ with $0 \leq \delta_{1}, \delta_{2}<q$, then by Lemma 2.5 $d_{q}\left(\tilde{A}_{x-1} C_{y}\right)-d_{q}\left(\tilde{A}_{x} C_{y-1}\right)=2\left(\gamma_{2}-\gamma_{1}\right)$; thus if $2 y-1>x$ we could replace the pair $\tilde{A}_{x-1} C_{y}$ by $\tilde{A}_{x} C_{y-1}$ without increasing $d_{q}(Z)$, so we may assume that $2 y \leq x+1$. Likewise if $2 y \leq x-2$ we could replace $\tilde{A}_{x-1} C_{y}$ by $\tilde{A}_{x-2} C_{y+1}$ (and then possibly adjust further the factors of type $A$ ), so we may assume $x-1 \leq 2 y$; thus we have $x-1 \leq 2 y \leq x+1$. If there are $A_{x}$ factors present (so that $z>0$ above), then we may argue similarly to deduce that $x \leq 2 y \leq x+2$; putting these conditions together we have $x \leq 2 y \leq x+1$, so that $y=\left\lceil\frac{x}{2}\right\rceil$. If there are no $A_{x}$ factors, then either $y=\left\lceil\frac{x}{2}\right\rceil$, or $2 y=x-1$; if the latter holds we may set $x^{\prime}=x-1$, $y^{\prime}=y$, and then we have $\left(\tilde{A}_{x^{\prime}}\right)^{s} C_{y^{\prime}}$ with $y^{\prime}=\frac{x^{\prime}}{2}$ (note that in this case, in which $z=s$ in the form for the type $A$ factors given above, we have $x^{\prime}$ even). Writing $a$ for $x$ (or $x^{\prime}$ ), we have $\ell=\operatorname{rank} Z=a z+(a-1)(s-z)+\left\lceil\frac{a}{2}\right\rceil+s=a s+\left\lceil\frac{a}{2}\right\rceil+z$, so $2 \ell=2 a s+2\left\lceil\frac{a}{2}\right\rceil+2 z=2 a s+a+\epsilon_{a}+2 z$ with $\epsilon_{a}+2 z<2 s+1$ (because if $z=s$, then $a$ is even). Therefore we have

$$
Z=\left(\tilde{A}_{a}\right)^{\left\lfloor\frac{b}{2}\right\rfloor}\left(\tilde{A}_{a-1}\right)^{s-\left\lfloor\frac{b}{2}\right\rfloor} C_{\left\lceil\frac{a}{2}\right\rceil} T_{s},
$$

where $2 \ell=a(2 s+1)+b$ with $0 \leq b<2 s+1$.

Having determined the form of $Z$, write $a=c q+d$ with $0 \leq d<q$, and apply the formulæ from Lemma 2.5(i,iii); we obtain

$$
\begin{aligned}
d_{q}(Z)= & \left\lfloor\frac{b}{2}\right\rfloor\left(c^{2} q+(2 c+1)(d+1)-1\right)+\left(s-\left\lfloor\frac{b}{2}\right\rfloor\right)\left(c^{2} q+(2 c+1) d-1\right) \\
& +\frac{1}{2} c^{2} q+(2 c+1)\left(\left\lceil\frac{a}{2}\right\rceil-\frac{1}{2} c q\right)+\epsilon_{q}\left\lceil\frac{c}{2}\right\rceil+s \\
= & c^{2} q\left(s+\frac{1}{2}\right)+(2 c+1)\left(d s+\left\lfloor\frac{b}{2}\right\rfloor+\left\lceil\frac{a}{2}\right\rceil-\frac{1}{2} c q\right)+\epsilon_{q}\left\lceil\frac{c}{2}\right\rceil \\
= & c^{2} q\left(s+\frac{1}{2}\right)+(2 c+1)\left(d s+\frac{a+b}{2}-\frac{1}{2} c q\right)+\epsilon_{q}\left\lceil\frac{c}{2}\right\rceil \\
= & c^{2} q\left(s+\frac{1}{2}\right)+(2 c+1)\left(\ell-c q\left(s+\frac{1}{2}\right)\right)+\epsilon_{q}\left\lceil\frac{c}{2}\right\rceil \\
= & (2 c+1) \ell-c(c+1) q\left(s+\frac{1}{2}\right)+\epsilon_{q}\left\lceil\frac{c}{2}\right\rceil \\
= & (2 c+1) \ell-\frac{1}{2} c(c+1) r+\epsilon_{r}\left\lceil\frac{c}{2}\right\rceil
\end{aligned}
$$

(note that the assumption that $n$ is odd means that $\epsilon_{q}=\epsilon_{r}$ ). As in the proof of Proposition 3.3. since we have $z r+e=2 \ell=a n+b=(c q+d) n+b=c r+(d n+b)$ 
with $d n+b \leq(q-1) n+(n-1)=r-1$, it follows that $c=z$, so

$$
d_{r}\left(C_{\ell}\right)=(2 z+1) \ell-\frac{1}{2} z(z+1) r+\epsilon_{r}\left\lceil\frac{z}{2}\right\rceil
$$

substituting from $2 \ell=z r+e$ gives $d_{r}\left(C_{\ell}\right)=d_{C_{\ell}, r}$ for $n$ odd, as required.

Now assume that $n$ is even and set $n=2 s$. Here there are two types of possibilities for the coefficients $b_{i}$, and thus for the centralizer $Z$, depending on whether $b_{0}=b_{\ell}=1$ or $b_{0}=b_{\ell}=0$; we shall need to consider each. First consider the possibility $b_{0}=b_{\ell}=1$; here there are $s-1$ nodes $i$ with $1 \leq i \leq \ell$ such that $b_{i}=1$, and so much as in the previous proposition we must have

$$
Z=\left(\tilde{A}_{a}\right)^{b}\left(\tilde{A}_{a-1}\right)^{s-b} T_{s}
$$

where $\ell=a s+b$ with $0 \leq b<s$. Again write $a=c q+d$ with $0 \leq d<q$, and apply the formulæ from Lemma 2.5(i); we obtain

$$
\begin{aligned}
d_{q}(Z) & =b\left(c^{2} q+(2 c+1)(d+1)-1\right)+(s-b)\left(c^{2} q+(2 c+1) d-1\right)+s \\
& =c^{2} q s+(2 c+1)(d s+b) \\
& =c^{2} q s+(2 c+1)(\ell-c q s) \\
& =(2 c+1) \ell-c(c+1) q s \\
& =(2 c+1) \ell-\frac{1}{2} c(c+1) r .
\end{aligned}
$$

Here we have $z r+e=2 \ell=a n+2 b=(c q+d) n+2 b=c r+d n+2 b$ with $d n+2 b \leq(q-1) n+2(s-1)=r-2$, so again we have $c=z$ and

$$
d_{q}(Z)=(2 z+1) \ell-\frac{1}{2} z(z+1) r
$$

substituting from $2 \ell=z r+e$ (and noting that $\epsilon_{r}=0$ here) gives $d_{q}(Z)=d_{C_{\ell}, r}$.

The second possibility here is that $b_{0}=b_{\ell}=0$; here there are $s$ nodes $i$ with $1 \leq i \leq \ell$ such that $b_{i}=1$. Again we must determine an optimal form for $Z$, which has $s-1$ factors of type $A$, two factors of type $C$ and an $(s-1)$-dimensional torus. As before we may assume that the type $A$ factors are $\left(\tilde{A}_{x}\right)^{z}\left(\tilde{A}_{x-1}\right)^{s-z}$, for some $x$ and some $0 \leq z<s$; the type $C$ factors likewise may be taken to be either $\left(C_{y}\right)^{2}$ or $C_{y} C_{y-1}$. Using the reasoning already given, if the type $C$ factors are of equal rank we must have either $y=\left\lceil\frac{x}{2}\right\rceil$, or $z=0$ and $2 y=x-1$; in the latter case we set $x^{\prime}=x-1, y^{\prime}=y$ and obtain $\left(\tilde{A}_{x^{\prime}}\right)^{s-1}\left(C_{y^{\prime}}\right)^{2}$ with $y^{\prime}=\frac{x^{\prime}}{2}($ and $z=s-1)$. On the other hand, if we have $C_{y} C_{y-1}$, then both $y$ and $y-1$ must satisfy these conditions; the only way this can happen is if we have $2 y=x+1$ with $z=0$, in which case we obtain $\left(\tilde{A}_{x-1}\right)^{s-1} C_{\left\lceil\frac{x}{2}\right\rceil} C_{\left\lceil\frac{x}{2}\right\rceil-1}$ with $x$ odd. Putting together these possibilities, writing $a$ for $x$ (or $x^{\prime}$ ) and equating $\operatorname{rank} Z$ to $\ell$, we find that we have

$$
Z=\left(\tilde{A}_{a}\right)^{b-\epsilon_{a}\left(1-\zeta_{b}\right)}\left(\tilde{A}_{a-1}\right)^{s-1-b+\epsilon_{a}\left(1-\zeta_{b}\right)} C_{\left\lceil\frac{a}{2}\right\rceil} C_{\left\lceil\frac{a}{2}\right\rceil-\epsilon_{a} \zeta_{b}} T_{s-1},
$$

where $\ell=a s+b$ with $0 \leq b<s$. 
As usual write $a=c q+d$ with $0 \leq d<q$, and apply the formulæ from Lemma 2.5(i), (iii); noting that $\epsilon_{q}=1$ because $n$ is even, we obtain

$$
\begin{aligned}
d_{q}(Z)= & \left(b-\epsilon_{a}\left(1-\zeta_{b}\right)\right)\left(c^{2} q+(2 c+1)(d+1)-1\right) \\
& +\left(s-1-b+\epsilon_{a}\left(1-\zeta_{b}\right)\right)\left(c^{2} q+(2 c+1) d-1\right) \\
& +\frac{1}{2} c^{2} q+(2 c+1)\left(\left\lceil\frac{a}{2}\right\rceil-\frac{1}{2} c q\right)+\epsilon_{q}\left\lceil\frac{c}{2}\right\rceil \\
& +\frac{1}{2} c^{2} q+(2 c+1)\left(\left\lceil\frac{a}{2}\right\rceil-\epsilon_{a} \zeta_{b}-\frac{1}{2} c q\right)+\epsilon_{q}\left\lceil\frac{c}{2}\right\rceil+s-1 \\
= & c^{2} q s+(2 c+1)\left(d(s-1)+b+2\left\lceil\frac{a}{2}\right\rceil-\epsilon_{a}-c q\right)+2\left\lceil\frac{c}{2}\right\rceil \\
= & c^{2} q s+(2 c+1)(\ell-c q s)+2\left\lceil\frac{c}{2}\right\rceil \\
= & (2 c+1) \ell-c(c+1) q s+2\left\lceil\frac{c}{2}\right\rceil \\
= & (2 c+1) \ell-\frac{1}{2} c(c+1) r+2\left\lceil\frac{c}{2}\right\rceil
\end{aligned}
$$

(observe that $2\left\lceil\frac{a}{2}\right\rceil-\epsilon_{a}=a$ ). As above we have $c=z$; this time however we have $d_{q}(Z)-d_{C_{\ell}, r}=2\left\lceil\frac{c}{2}\right\rceil \geq 0$. Combining the two types of possibilities, we conclude that $d_{r}\left(C_{\ell}\right)=d_{C_{\ell}, r}$ for $n$ even, as required. This completes the proof.

Proposition 3.5. With the notation established, $d_{r}\left(D_{\ell}\right)=d_{D_{\ell}, r}$.

Proof. We have $h=2 \ell-2$; and $m_{i}=2$ for all $2 \leq i \leq \ell-2$ while $m_{0}=m_{1}=$ $m_{\ell-1}=m_{\ell}=1$. Again we shall need to argue separately for $n$ odd and $n$ even; first assume $n$ is odd and set $n=2 s+1$. By Lemma 3.2 there are two types of possibilities for the coefficients $b_{i}$, and hence the centralizer $Z$, according to whether the number of $b_{i}$ equal to 1 for which $i \in\{0,1, \ell-1, \ell\}$ is 1 or 3 ; we shall consider each. First assume that the $b_{i}$ which are equal to 1 correspond to $s-1$ nodes $i$ with $2 \leq i \leq \ell-2$, together with three of the four nodes $0,1, \ell-1$ and $\ell$; this means that $Z$ has $s$ factors of type $A$ and an $(s+1)$-dimensional torus. In similar fashion to the above, we see that we must have

$$
Z=\left(A_{a}\right)^{b}\left(A_{a-1}\right)^{s-b} T_{s+1},
$$

where $\ell-1=a s+b$ with $0 \leq b<s$. As usual write $a=c q+d$ with $0 \leq d<q$, and apply the formulæ from Lemma 2.5(i); we obtain

$$
\begin{aligned}
d_{q}(Z) & =b\left(c^{2} q+(2 c+1)(d+1)-1\right)+(s-b)\left(c^{2} q+(2 c+1) d-1\right)+s+1 \\
& =c^{2} q s+(2 c+1)(d s+b)+1 \\
& =c^{2} q s+(2 c+1)(\ell-1-c q s)+1 \\
& =(2 c+1) \ell-c(c+1) q s-2 c \\
& =(2 c+1) \ell-\frac{1}{2} c(c+1) q(n-1)-2 c \\
& =(2 c+1) \ell-\frac{1}{2} c(c+1)(r-q)-2 c .
\end{aligned}
$$

Here we have $z r+e=2 \ell-2=a(n-1)+2 b=(c q+d)(n-1)+2 b=c(r-q)+$ $d(n-1)+2 b$ with $d(n-1)+2 b \leq(q-1)(n-1)+2(s-1)=r-q-2$; thus if we set $f=d(n-1)+2 b$, then we have $z r+e=c(r-q)+f$ with $0 \leq f<r-q$. It follows that $c \geq z$, and we find that

$$
\begin{aligned}
d_{q}(Z)-d_{D_{\ell}, r}= & (c-z)\left(f+\frac{1}{2}(r-q)(c-z-1)\right)+\frac{1}{2} z((z+1) q-3) \\
& +\left(\frac{z}{2}-\epsilon_{r}\left\lceil\frac{z}{2}\right\rceil+\epsilon_{z}\right) \\
\geq & 0
\end{aligned}
$$

(note that $\epsilon_{r}=\epsilon_{q}$ as $n$ is odd, and thus if $z=q=1$ the second term is $-\frac{1}{2}$ but the third is $\left.\frac{1}{2}\right)$. 
We now consider the other type of possibility for $Z$. Here we assume that the $b_{i}$ which are equal to 1 correspond to $s$ nodes $i$ with $2 \leq i \leq \ell-2$, together with one of the four nodes $0,1, \ell-1$ and $\ell$; this means that $Z$ has $s$ factors of type $A$, one factor of type $D$ and an $s$-dimensional torus. We shall again need to determine a configuration which minimizes $d_{q}(Z)$.

As before, the type $A$ factors may be assumed to take the form $\left(A_{x}\right)^{z}\left(A_{x-1}\right)^{s-z}$, for some $x$ and some $0 \leq z<s$; suppose the type $D$ factor is $D_{y}$. If we write $x=\gamma_{1} q+\delta_{1}$ and $2 y-2=\gamma_{2} q+\delta_{2}$ with $0 \leq \delta_{1}, \delta_{2}<q$, then by Lemma 2.5 $d_{q}\left(A_{x-1} D_{y}\right)-d_{q}\left(A_{x} D_{y-1}\right)=2\left(\gamma_{2}-\gamma_{1}-\epsilon_{\gamma_{2}} \zeta_{\delta_{2}}\right)$; thus if $2 y-2>x$ we could replace the pair $A_{x-1} D_{y}$ by $A_{x} D_{y-1}$ without increasing $d_{q}(Z)$ (note that in this case if $\gamma_{2}=\gamma_{1}$, then $\delta_{2}>0$ and so $\zeta_{\delta_{2}}=0$ ), so we may assume that $2 y \leq x+2$. Likewise if $2 y \leq x-1$ we could replace $A_{x-1} D_{y}$ by $A_{x-2} D_{y+1}$ (and then possibly adjust further the factors of type $A$ ), so we may assume $x \leq 2 y$; thus we have $x \leq 2 y \leq x+2$. The remainder of the argument is exactly similar to that in the proof of Proposition 3.4, and we conclude that we have

$$
Z=\left(A_{a}\right)^{\left\lfloor\frac{b}{2}\right\rfloor}\left(A_{a-1}\right)^{s-\left\lfloor\frac{b}{2}\right\rfloor} D_{\left\lceil\frac{a+1}{2}\right\rceil} T_{s},
$$

where $2 \ell-1=a(2 s+1)+b$ with $0 \leq b<2 s+1$.

Having determined the form of $Z$, write $a=c q+d$ with $0 \leq d<q$, and apply the formulæ from Lemma 2.5)(i),(iv); we obtain

$$
\begin{aligned}
d_{q}(Z)= & \left\lfloor\frac{b}{2}\right\rfloor\left(c^{2} q+(2 c+1)(d+1)-1\right)+\left(s-\left\lfloor\frac{b}{2}\right\rfloor\right)\left(c^{2} q+(2 c+1) d-1\right) \\
& +\frac{1}{2} c^{2} q+(2 c+1)\left(\left\lceil\frac{a+1}{2}\right\rceil-\frac{1}{2} c q\right)+\epsilon_{q}\left\lceil\frac{c}{2}\right\rceil-c-\epsilon_{c}+s \\
= & c^{2} q\left(s+\frac{1}{2}\right)+(2 c+1)\left(d s+\left\lfloor\frac{b}{2}\right\rfloor\left\lceil\frac{a+1}{2}\right\rceil-\frac{1}{2} c q\right)+\epsilon_{q}\left\lceil\frac{c}{2}\right\rceil-c-\epsilon_{c} \\
= & c^{2} q\left(s+\frac{1}{2}\right)+(2 c+1)\left(d s+\frac{a+b+1}{2}-\frac{1}{2} c q\right)+\epsilon_{q}\left\lceil\frac{c}{2}\right\rceil-c-\epsilon_{c} \\
= & c^{2} q\left(s+\frac{1}{2}\right)+(2 c+1)\left(\ell-c q\left(s+\frac{1}{2}\right)\right)+\epsilon_{q}\left\lceil\frac{c}{2}\right\rceil-c-\epsilon_{c} \\
= & (2 c+1) \ell-c(c+1) q\left(s+\frac{1}{2}\right)+\epsilon_{q}\left\lceil\frac{c}{2}\right\rceil-c-\epsilon_{c} \\
= & (2 c+1) \ell-\frac{1}{2} c(c+1) r+\epsilon_{r}\left\lceil\frac{c}{2}\right\rceil-c-\epsilon_{c}
\end{aligned}
$$

(note once more that the assumption that $n$ is odd means that $\epsilon_{q}=\epsilon_{r}$ ). Here we have $z r+e=2 \ell-2=a n+b-1=(c q+d) n+b-1=c r+(d n+b-1)$ with $d n+b \leq(q-1) n+(n-1)=r-1$. Thus either $c=z$, or we must have $c=z+1$ and $2 \ell-1=(z+1) r$; but if the latter holds then, using the fact that $c$ and $r$ are both odd, we see that $(2 c+1) \ell-\frac{1}{2} c(c+1) r+\epsilon_{r}\left\lceil\frac{c}{2}\right\rceil-c-\epsilon_{c}=(2 z+1) \ell-\frac{1}{2} z(z+1) r+\epsilon_{r}\left\lceil\frac{z}{2}\right\rceil-z-\epsilon_{z}$ anyway. Thus we have

$$
d_{q}(Z)=(2 z+1) \ell-\frac{1}{2} z(z+1) r+\epsilon_{r}\left\lceil\frac{z}{2}\right\rceil-z-\epsilon_{z}=d_{D_{\ell}, r}
$$

so combining the two possibilities we have $d_{r}\left(D_{\ell}\right)=d_{D_{\ell}, r}$ for $n$ odd, as required.

Now assume $n$ is even and set $n=2 s$. Here the number of $b_{i}$ equal to 1 for which $i \in\{0,1, \ell-1, \ell\}$ must be even, and thus can be 0,2 or 4 ; this gives a number of possibilities for the type of $Z$. If $b_{i}=1$ for all $i \in\{0,1, \ell-1, \ell\}$, there must be $s-1$ factors of type $A$ and an $(s+1)$-dimensional torus; we find that

$$
Z=\left(A_{a}\right)^{b}\left(A_{a-1}\right)^{s-1-b} T_{s+1}
$$


where $\ell-2=a(s-1)+b$ with $0 \leq b<s-1$. Writing $a=c q+d$ with $0 \leq d<q$ in the usual fashion, we obtain

$$
\begin{aligned}
d_{q}(Z) & =b\left(c^{2} q+(2 c+1)(d+1)-1\right)+(s-1-b)\left(c^{2} q+(2 c+1) d-1\right)+s+1 \\
& =c^{2} q(s-1)+(2 c+1)(d(s-1)+b)+2 \\
& =c^{2} q(s-1)+(2 c+1)(\ell-2-c q(s-1))+2 \\
& =(2 c+1) \ell-c(c+1) q(s-1)-4 c \\
& =(2 c+1) \ell-\frac{1}{2} c(c+1) q(n-2)-4 c \\
& =(2 c+1) \ell-\frac{1}{2} c(c+1)(r-2 q)-4 c .
\end{aligned}
$$

We have $z r+e=2 \ell-2=a(n-2)+2 b+2=c(r-2 q)+d(n-2)+2 b+2$, and $d(n-2)+2 b+2 \leq(q-1)(n-2)+2(s-2)+2=r-2 q$; thus $c \geq z$, and if we set $f=d(n-2)+2 b$ we find that

$$
d_{q}(Z)-d_{D_{\ell}, r}=(c-z)\left(f+\frac{1}{2}(r-2 q)(c-z-1)\right)+z((z+1) q-3)+\epsilon_{z} \geq 0
$$

(note that if $z=q=1$, then the second term is -1 but the third is 1 ).

Next assume that the number of $b_{i}$ equal to 1 for which $i \in\{0,1, \ell-1, \ell\}$ is 2 ; here we must subdivide yet further, because the two nodes concerned might be at different ends of the extended Dynkin diagram or at the same end. If they are at different ends, then $Z$ has $s$ factors of type $A$ and an $s$-dimensional torus, and we obtain

$$
Z=\left(A_{a}\right)^{b}\left(A_{a-1}\right)^{s-b} T_{s},
$$

where $\ell=a s+b$ with $0 \leq b<s$. Setting $a=c q+d$ with $0 \leq d<q$ we obtain

$$
\begin{aligned}
d_{q}(Z) & =b\left(c^{2} q+(2 c+1)(d+1)-1\right)+(s-b)\left(c^{2} q+(2 c+1) d-1\right)+s \\
& =c^{2} q(s-1)+(2 c+1)(d s+b) \\
& =c^{2} q(s-1)+(2 c+1)(\ell-c q s) \\
& =(2 c+1) \ell-c(c+1) q s \\
& =(2 c+1) \ell-\frac{1}{2} c(c+1) r .
\end{aligned}
$$

Here we have $z r+e=2 \ell-2=a n+2 b-2=c r+d n+2 b-2$, and $d n+2 b-2 \leq$ $(q-1) n+2(s-1)-2=r-4$. Thus either $c=z$, or we must have $c=z+1$ and $2 \ell=(z+1) r$; but if the latter holds then we see that $(2 c+1) \ell-\frac{1}{2} c(c+1) r=$ $(2 z+1) \ell-\frac{1}{2} z(z+1) r$ anyway. Thus in either case we have

$$
\begin{aligned}
d_{q}(Z)-d_{D_{\ell}, r} & =(2 z+1) \ell-\frac{1}{2} z(z+1) r-\frac{1}{2} z^{2} r-\frac{1}{2} e(2 z+1)-\epsilon_{r}\left\lceil\frac{z}{2}\right\rceil-z-1+\epsilon_{z} \\
& =z+\epsilon_{z} \\
& \geq 0
\end{aligned}
$$

If however the two nodes are at the same end of the extended Dynkin diagram, then $Z$ has $s-1$ factors of type $A$, one factor of type $D$ and an $s$-dimensional torus. Proceeding in a similar fashion to the case above with $n$ odd, we find that

$$
Z=\left(A_{a}\right)^{\left\lfloor\frac{b}{2}\right\rfloor}\left(A_{a-1}\right)^{s-1-\left\lfloor\frac{b}{2}\right\rfloor} D_{\left\lceil\frac{a+1}{2}\right\rceil} T_{s},
$$


where $2 \ell-3=a(2 s-1)+b$ with $0 \leq b<2 s-1$. Setting $a=c q+d$ with $0 \leq d<q$ we obtain

$$
\begin{aligned}
d_{q}(Z)= & \left\lfloor\frac{b}{2}\right\rfloor\left(c^{2} q+(2 c+1)(d+1)-1\right)+\left(s-1-\left\lfloor\frac{b}{2}\right\rfloor\right)\left(c^{2} q+(2 c+1) d-1\right) \\
& +\frac{1}{2} c^{2} q+(2 c+1)\left(\left\lceil\frac{a+1}{2}\right\rceil-\frac{1}{2} c q\right)+\epsilon_{q}\left\lceil\frac{c}{2}\right\rceil-c-\epsilon_{c}+s \\
= & c^{2} q\left(s-\frac{1}{2}\right)+(2 c+1)\left(d(s-1)+\left\lfloor\frac{b}{2}\right\rfloor+\left\lceil\frac{a+1}{2}\right\rceil-\frac{1}{2} c q\right)+\left\lceil\frac{c}{2}\right\rceil-c-\epsilon_{c}+1 \\
= & c^{2} q\left(s-\frac{1}{2}\right)+(2 c+1)\left(d(s-1)+\frac{a+b+1}{2}-\frac{1}{2} c q\right)+\left\lceil\frac{c}{2}\right\rceil-c-\epsilon_{c}+1 \\
= & c^{2} q\left(s-\frac{1}{2}\right)+(2 c+1)\left(\ell-1-c q\left(s-\frac{1}{2}\right)\right)+\left\lceil\frac{c}{2}\right\rceil-c-\epsilon_{c}+1 \\
= & (2 c+1) \ell-c(c+1) q\left(s-\frac{1}{2}\right)+\left\lceil\frac{c}{2}\right\rceil-3 c-\epsilon_{c} \\
= & (2 c+1) \ell-\frac{1}{2} c(c+1)(r-q)+\left\lceil\frac{c}{2}\right\rceil-3 c-\epsilon_{c} .
\end{aligned}
$$

Here we have $z r+e=2 \ell-2=a(n-1)+b+1=c(r-q)+d(n-1)+b+1$, and $d(n-1)+b+1 \leq(q-1)(n-1)+2 s-2+1=r-q$; thus $c \geq z$, and if we set $f=d(n-1)+b$ we find that

$$
\begin{aligned}
d_{q}(Z)-d_{D_{\ell}, r}= & (c-z)\left(f+\frac{1}{2}(r-q)(c-z-1)\right)+\frac{1}{2} z((z+1) q-3) \\
& +\left(\frac{1}{2}(c-z)+\epsilon_{z}-\frac{1}{2} \epsilon_{c}\right) \\
\geq & 0
\end{aligned}
$$

(note again that if $z=q=1$, then the second term is $-\frac{1}{2}$ but the third is at least $\left.\frac{1}{2}\right)$.

Finally we must consider the possibility that $b_{i}=0$ for all $i \in\{0,1, \ell-1, \ell\}$; here $Z$ has $s-1$ factors of type $A$, two of type $D$ and an $(s-1)$-dimensional torus. As usual the type $A$ factors may be taken to be $\left(A_{x}\right)^{z}\left(A_{x-1}\right)^{s-z}$, for some $x$ and some $0 \leq z<s$, while the type $D$ factors may be taken to be either $\left(D_{y}\right)^{2}$ or $D_{y} D_{y-1}$ for some $y$. Arguing in precisely similar fashion to the analogous case in Proposition 3.4 shows that

$$
Z=\left(A_{a}\right)^{b-\epsilon_{a+1}\left(1-\zeta_{b}\right)}\left(A_{a-1}\right)^{s-1-b+\epsilon_{a+1}\left(1-\zeta_{b}\right)} D_{\left\lceil\frac{a+1}{2}\right\rceil} D_{\left\lceil\frac{a+1}{2}\right\rceil-\epsilon_{a+1} \zeta_{b}} T_{s-1},
$$

where $\ell-1=a s+b$ with $0 \leq b<s$. As usual, write $a=c q+d$ with $0 \leq d<q$ and apply the formulæ from Lemma 2.5(i), (iv); noting that $\epsilon_{q}=1$ because $n$ is even, and that if $d=0$, then $c(a+1)$ is even because $a=c q \equiv c \bmod 2$, we obtain

$$
\begin{aligned}
d_{q}(Z)= & \left(b-\epsilon_{a+1}\left(1-\zeta_{b}\right)\right)\left(c^{2} q+(2 c+1)(d+1)-1\right) \\
& +\left(s-1-b+\epsilon_{a+1}\left(1-\zeta_{b}\right)\right)\left(c^{2} q+(2 c+1) d-1\right) \\
& +\frac{1}{2} c^{2} q+(2 c+1)\left(\left\lceil\frac{a+1}{2}\right\rceil-\frac{1}{2} c q\right)+\epsilon_{q}\left\lceil\frac{c}{2}\right\rceil-c-\epsilon_{c} \\
& +\frac{1}{2} c^{2} q+(2 c+1)\left(\left\lceil\frac{a+1}{2}\right\rceil-\epsilon_{a+1} \zeta_{b}-\frac{1}{2} c q\right)+\epsilon_{q}\left\lceil\frac{c}{2}\right\rceil-c-\epsilon_{c}+2 \epsilon_{c(a+1)} \zeta_{d} \zeta_{b} \\
& +s-1 \\
= & c^{2} q s+(2 c+1)\left(d(s-1)+b+2\left\lceil\frac{a+1}{2}\right\rceil-\epsilon_{a+1}-c q\right)+2\left\lceil\frac{c}{2}\right\rceil-2 c-2 \epsilon_{c} \\
= & c^{2} q s+(2 c+1)(\ell-c q s)-c-\epsilon_{c} \\
= & (2 c+1) \ell-c(c+1) q s-c-\epsilon_{c} \\
= & (2 c+1) \ell-\frac{1}{2} c(c+1) r-c-\epsilon_{c} .
\end{aligned}
$$

Here $z r+e=2 \ell-2=a n+2 b=c r+d n+2 b$ and $d n+2 b \leq(q-1) n+2(s-1)=r-2$, so $c=z$, and we have

$$
d_{q}(Z)=(2 z+1) \ell-\frac{1}{2} z(z+1) r-z-\epsilon_{z}=d_{D_{\ell}, r}
$$


(note that $\epsilon_{r}=0$ as $n$ is even here). Combining all the possibilities we conclude that $d_{r}\left(D_{\ell}\right)=d_{D_{\ell}, r}$ for $n$ even, as required. This completes the proof.

Proposition 3.6. With the notation established, $d_{r}\left(B_{\ell}\right)=d_{B_{\ell}, r}$.

Proof. We have $h=2 \ell$; and $m_{i}=2$ for all $2 \leq i \leq \ell$ while $m_{0}=m_{1}=1$. Yet again we shall need to argue separately for $n$ odd and $n$ even; first assume $n$ is odd and set $n=2 s+1$. By Lemma 3.2 and the fact that $\alpha_{\ell}$ is short, there are two types of possibilities for the coefficients $b_{i}$, and hence $Z$, according as $b_{\ell}=0$ or 1 . First assume that $b_{\ell}=1$. Then $Z$ has $s$ factors of type $A$ and an $s$-dimensional torus, and we have

$$
Z=\left(A_{a}\right)^{b}\left(A_{a-1}\right)^{s-b} T_{s}
$$

where $\ell=a s+b$ with $0 \leq b<s$. Setting $a=c q+d$ with $0 \leq d<q$ we obtain

$$
\begin{aligned}
d_{q}(Z) & =b\left(c^{2} q+(2 c+1)(d+1)-1\right)+(s-b)\left(c^{2} q+(2 c+1) d-1\right)+s \\
& =c^{2} q s+(2 c+1)(d s+b) \\
& =c^{2} q s+(2 c+1)(\ell-c q s) \\
& =(2 c+1) \ell-c(c+1) q s \\
& =(2 c+1) \ell-\frac{1}{2} c(c+1)(r-q) .
\end{aligned}
$$

Here $z r+e=2 \ell=a(n-1)+2 b=c(r-q)+d(n-1)+2 b$, and $d(n-1)+2 b \leq$ $(q-1)(n-1)+2(s-1)=(r-q)-2$; thus $c \geq z$, and if we set $f=d(n-1)+2 b$ we find that

$$
\begin{aligned}
d_{q}(Z)-d_{B_{\ell}, r} & =(c-z)\left(f+\frac{1}{2}(r-q)(c-z-1)\right)+\frac{1}{2} z((z+1) q-2)+\left(z-\epsilon_{r}\left\lceil\frac{z}{2}\right\rceil\right) \\
& \geq 0 .
\end{aligned}
$$

Now assume instead that $b_{\ell}=0$. Then $Z$ has $s$ factors of type $A$, one of type $B$ and an $s$-dimensional torus. Arguing precisely as in the proof of Proposition 3.4 we see that

$$
Z=\left(A_{a}\right)^{\left\lfloor\frac{b}{2}\right\rfloor}\left(A_{a-1}\right)^{s-\left\lfloor\frac{b}{2}\right\rfloor} B_{\left\lceil\frac{a}{2}\right\rceil} T_{s},
$$

where $2 \ell=a(2 s+1)+b$ with $0 \leq b<2 s+1$. On setting $a=c q+d$ with $0 \leq d<q$, the calculation for $d_{q}(Z)$ is identical to that given in the proof of Proposition 3.4 for $n$ odd, and gives

$$
d_{q}(Z)=(2 c+1) \ell-\frac{1}{2} c(c+1) r+\epsilon_{r}\left\lceil\frac{c}{2}\right\rceil .
$$

Since $z r+e=2 \ell=a n+b=c r+d n+b$ with $d n+b \leq(q-1) n+(n-1)=r-1$, we have $c=z$ and thus

$$
d_{q}(Z)=(2 z+1) \ell-\frac{1}{2} z(z+1) r+\epsilon_{r}\left\lceil\frac{z}{2}\right\rceil=d_{B_{\ell}, r} .
$$

Combining the two possibilities we see that $d_{r}\left(B_{\ell}\right)=d_{B_{\ell}, r}$ for $n$ odd, as required.

Now assume that $n$ is even, and set $n=2 s$. Here either $b_{0}=b_{1}=0$ or $b_{0}=b_{1}=1$, and $b_{\ell}=0$ or 1 ; so there are four types of possibility for $Z$. First take the case where $b_{0}=b_{1}=b_{\ell}=1$. Here $Z$ has $s-1$ factors of type $A$ and an $s$-dimensional torus, and we obtain

$$
Z=\left(A_{a}\right)^{b}\left(A_{a-1}\right)^{s-1-b} T_{s},
$$


where $\ell-1=a(s-1)+b$ with $0 \leq b<s-1$. Setting $a=c q+d$ with $0 \leq d<q$ gives

$$
\begin{aligned}
d_{q}(Z) & =b\left(c^{2} q+(2 c+1)(d+1)-1\right)+(s-1-b)\left(c^{2} q+(2 c+1) d-1\right)+s \\
& =c^{2} q(s-1)+(2 c+1)(d(s-1)+b)+1 \\
& =c^{2} q(s-1)+(2 c+1)(\ell-1-c q(s-1))+1 \\
& =(2 c+1) \ell-c(c+1) q(s-1)-2 c \\
& =(2 c+1) \ell-\frac{1}{2} c(c+1)(r-2 q)-2 c .
\end{aligned}
$$

Here $z r+e=2 \ell=a(n-2)+2 b+2=c(r-2 q)+d(n-2)+2 b+2$, and $d(n-2)+2 b+2 \leq(q-1)(n-2)+2(s-2)+2=r-2 q$. Thus $c \geq z$, and if we set $f=d(n-2)+2 b$ we find that

$$
d_{q}(Z)-d_{B_{\ell}, r}=(c-z)\left(f+\frac{1}{2}(r-2 q)(c-z-1)\right)+z((z+1) q-2) \geq 0 .
$$

Next consider $b_{0}=b_{1}=1, b_{\ell}=0$. Here $Z$ has $s-1$ factors of type $A$, one factor of type $B$ and an $s$-dimensional torus, and arguing as before we find

$$
Z=\left(A_{a}\right)^{\left\lfloor\frac{b}{2}\right\rfloor}\left(A_{a-1}\right)^{s-1-\left\lfloor\frac{b}{2}\right\rfloor} B_{\left\lceil\frac{a}{2}\right\rceil} T_{s},
$$

where $2 \ell-2=a(2 s-1)+b$ with $0 \leq b<2 s-1$. Write $a=c q+d$ with $0 \leq d<q$. Noting that $\epsilon_{q}=1$ here and using the formulæ from Lemma 2.5(i),(ii) we obtain

$$
\begin{aligned}
d_{q}(Z)= & \left\lfloor\frac{b}{2}\right\rfloor\left(c^{2} q+(2 c+1)(d+1)-1\right)+\left(s-1-\left\lfloor\frac{b}{2}\right\rfloor\right)\left(c^{2} q+(2 c+1) d-1\right) \\
& +\frac{1}{2} c^{2} q+(2 c+1)\left(\left\lceil\frac{a}{2}\right\rceil-\frac{1}{2} c q\right)+\epsilon_{q}\left\lceil\frac{c}{2}\right\rceil+s \\
= & c^{2} q\left(s-\frac{1}{2}\right)+(2 c+1)\left(d(s-1)+\left\lfloor\frac{b}{2}\right\rfloor+\left\lceil\frac{a}{2}\right\rceil-\frac{1}{2} c q\right)+\left\lceil\frac{c}{2}\right\rceil+1 \\
= & c^{2} q\left(s-\frac{1}{2}\right)+(2 c+1)\left(d(s-1)+\frac{a+b}{2}-\frac{1}{2} c q\right)+\left\lceil\frac{c}{2}\right\rceil+1 \\
= & c^{2} q\left(s-\frac{1}{2}\right)+(2 c+1)\left(\ell-1-c q\left(s-\frac{1}{2}\right)\right)+\left\lceil\frac{c}{2}\right\rceil+1 \\
= & (2 c+1) \ell-c(c+1) q\left(s-\frac{1}{2}\right)+\left\lceil\frac{c}{2}\right\rceil-2 c \\
= & (2 c+1) \ell-\frac{1}{2} c(c+1)(r-q)+\left\lceil\frac{c}{2}\right\rceil-2 c .
\end{aligned}
$$

Here $z r+e=2 \ell=a(n-1)+b+2=c(r-q)+d(n-1)+b+2$, with $d(n-1)+b+2 \leq$ $(q-1)(n-1)+(2 s-2)+2=r-q+1$. Thus $z r+e \leq(c+1)(r-q)+1$. If we were to have $c<z$ this would give $z r+e \leq z(r-q)+1$, or $e \leq 1-z q$, which would force $e=0, z=q=1$, whence $2 \ell=r=n$, contrary to our assumption that $n<h$. Therefore we have $c \geq z$, and if we set $f=d(n-1)+b$ we find that

$$
\begin{aligned}
d_{q}(Z)-d_{B_{\ell}, r}= & (c-z)\left(f+\frac{1}{2}(r-q)(c-z-1)\right)+\frac{1}{2} z((z+1) q-3) \\
& +\frac{1}{2}\left((c-z)+\epsilon_{c}\right) \\
\geq & 0
\end{aligned}
$$

(again note that if $z=q=1$, then the second term is $-\frac{1}{2}$ but the third is at least $\left.\frac{1}{2}\right)$.

Now consider the case $b_{0}=b_{1}=0, b_{\ell}=1$. Here $Z$ has $s-1$ factors of type $A$, one factor of type $D$ and an $(s-1)$-dimensional torus, and we find

$$
Z=\left(A_{a}\right)^{\left\lfloor\frac{b}{2}\right\rfloor}\left(A_{a-1}\right)^{s-1-\left\lfloor\frac{b}{2}\right\rfloor} D_{\left\lceil\frac{a+1}{2}\right\rceil} T_{s-1},
$$


where $2 \ell-1=a(2 s-1)+b$ with $0 \leq b<2 s-1$. Setting $a=c q+d$ with $0 \leq d<q$ and recalling that $\epsilon_{q}=1$ we obtain

$$
\begin{aligned}
d_{q}(Z)= & \left\lfloor\frac{b}{2}\right\rfloor\left(c^{2} q+(2 c+1)(d+1)-1\right)+\left(s-1-\left\lfloor\frac{b}{2}\right\rfloor\right)\left(c^{2} q+(2 c+1) d-1\right) \\
& +\frac{1}{2} c^{2} q+(2 c+1)\left(\left\lceil\frac{a+1}{2}\right\rceil-\frac{1}{2} c q\right)+\epsilon_{q}\left\lceil\frac{c}{2}\right\rceil-c-\epsilon_{c}+s-1 \\
= & c^{2} q\left(s-\frac{1}{2}\right)+(2 c+1)\left(d(s-1)+\left\lfloor\frac{b}{2}\right\rfloor+\left\lceil\frac{a+1}{2}\right\rceil-\frac{1}{2} c q\right)+\left\lceil\frac{c}{2}\right\rceil-c-\epsilon_{c} \\
= & c^{2} q\left(s-\frac{1}{2}\right)+(2 c+1)\left(d(s-1)+\frac{a+b+1}{2}-\frac{1}{2} c q\right)-\left\lceil\frac{c}{2}\right\rceil \\
= & c^{2} q\left(s-\frac{1}{2}\right)+(2 c+1)\left(\ell-c q\left(s-\frac{1}{2}\right)\right)-\left\lceil\frac{c}{2}\right\rceil \\
= & (2 c+1) \ell-c(c+1) q\left(s-\frac{1}{2}\right)-\left\lceil\frac{c}{2}\right\rceil \\
= & (2 c+1) \ell-\frac{1}{2} c(c+1)(r-q)-\left\lceil\frac{c}{2}\right\rceil .
\end{aligned}
$$

Here we have $z r+e=2 \ell=a(n-1)+b+1=c(r-q)+d(n-1)+b+1$, and $d(n-1)+b+1 \leq(q-1)(n-1)+2 s-2+1=r-q$. Thus $c \geq z$, and if we set $f=d(n-1)+b$ we find that

$d_{q}(Z)-d_{B_{\ell}, r}=(c-z)\left(f+\frac{1}{2}(r-q)(c-z-1)\right)+\frac{1}{2} z((z+1) q-2)+\frac{1}{2}\left(c-\epsilon_{c}\right) \geq 0$.

Finally we must consider the case $b_{0}=b_{1}=b_{\ell}=0$. Here $Z$ has $s-1$ factors of type $A$, one factor of type $B$, one factor of type $D$ and an $(s-1)$-dimensional torus. Again we must determine an optimal configuration. As usual we may take the type $A$ factors to be $\left(A_{x}\right)^{z}\left(A_{x-1}\right)^{s-1-z}$ for some $x$ and some $0 \leq z<s-1$, and easy considerations show that the factors of types $B$ and $D$ may be either $B_{y} D_{y}$ or $B_{y-1} D_{y}$ for some $y$. Using the reasoning already given for comparing factors of type $A$ with those of type $B$ or $D$, we see that if we have $B_{y} D_{y}$, then either $2 y=x+1$, or $z=0$ and $2 y=x$. If the latter holds we set $x^{\prime}=x-1, y^{\prime}=y$ and obtain $\left(A_{x^{\prime}}\right)^{s-1} B_{y^{\prime}} D_{y^{\prime}}$ with $2 y^{\prime}=x^{\prime}+1$. Likewise if we have $B_{y-1} D_{y}$, then either $2 y=x+2$, or $z=0$ and $2 y=x+1$. Again, if the latter holds we set $x^{\prime}=x-1$, $y^{\prime}=y$ and obtain $\left(A_{x^{\prime}}\right)^{s-1} B_{y^{\prime}-1} D_{y^{\prime}}$ with $2 y^{\prime}=x^{\prime}+2$. Putting all of these together we see that we have

$$
Z=\left(A_{a}\right)^{b}\left(A_{a-1}\right)^{s-1-b} B_{\left\lceil\frac{a}{2}\right\rceil} D_{\left\lceil\frac{a+1}{2}\right\rceil} T_{s-1},
$$

where $\ell-1=a s+b$ with $0 \leq b<s$. As usual write $a=c q+d$ with $0 \leq d<q$; using the formulæ in Lemma 2.5 (i), (ii), (iv) and noting that $\epsilon_{q}=1$ here we obtain

$$
\begin{aligned}
d_{q}(Z)= & b\left(c^{2} q+(2 c+1)(d+1)-1\right)+(s-1-b)\left(c^{2} q+(2 c+1) d-1\right) \\
& +\frac{1}{2} c^{2} q+(2 c+1)\left(\left\lceil\frac{a}{2}\right\rceil-\frac{1}{2} c q\right)+\epsilon_{q}\left\lceil\frac{c}{2}\right\rceil \\
& +\frac{1}{2} c^{2} q+(2 c+1)\left(\left\lceil\frac{a+1}{2}\right\rceil-\frac{1}{2} c q\right)+\epsilon_{q}\left\lceil\frac{c}{2}\right\rceil-c-\epsilon_{c}+s-1 \\
= & c^{2} q s+(2 c+1)\left(d(s-1)+b+\left\lceil\frac{a}{2}\right\rceil+\left\lceil\frac{a+1}{2}\right\rceil-c q\right)+2\left\lceil\frac{c}{2}\right\rceil-c-\epsilon_{c} \\
= & c^{2} q s+(2 c+1)(d(s-1)+b+a+1-c q) \\
= & c^{2} q s+(2 c+1)(\ell-c q s) \\
= & (2 c+1) \ell-c(c+1) q s \\
= & (2 c+1) \ell-\frac{1}{2} c(c+1) r .
\end{aligned}
$$

Here $z r+e=2 \ell=a n+2 b+2=c r+d n+2 b+2$, with $d n+2 b+2 \leq(q-1) n+$ $2(s-1)+2=r$. Thus either $c=z$, or we must have $c=z-1$ and $2 \ell=z r$; but if the latter holds, then we see that $(2 c+1) \ell-\frac{1}{2} c(c+1) r=(2 z+1) \ell-\frac{1}{2} z(z+1) r$ anyway. Thus in either case we have

$$
d_{q}(Z)=(2 z+1) \ell-\frac{1}{2} z(z+1) r=d_{B_{\ell}, r}
$$


(note that $\epsilon_{r}=0$ as $n$ is even here). Combining all possibilities we see that $d_{r}\left(B_{\ell}\right)=d_{B_{\ell}, r}$ for $n$ even, as required. This completes the proof.

Proposition 3.7. With the notation established, if $G$ has root system $\Phi$ of exceptional type, then $d_{r}(G)=d_{\Phi, r}$.

Proof. We begin by considering the cases in which $r<h$. Here we proceed by inspection, using the same general strategy as in the classical cases: given $n$, Lemma 3.2 is used to restrict the possibilities for $Z$ which must be considered, and then the formulæ of Lemmas [2.5 and 2.7 are used to calculate $d_{q}(Z)$ for each $Z$. For convenience, we present the relevant information in tabular form: given $\Phi$, for each $r<h$ we give the value $d_{\Phi, r}$ and various pairs $(p, Z)$, one for each prime $p$ dividing $r$ and then finally one for an arbitrary $p$ coprime to $r$, such that $Z$ is a centralizer of a semisimple element of order $n$ with minimal value of $d_{q}(Z)$. (Note that if $\Phi$ is of type $E_{7}$ or $E_{8}$, the standard notation is used to distinguish between isomorphic but non-conjugate subsystems: in $E_{7}$ an $\left(A_{1}^{3}\right)^{\prime}$ subsystem is one lying in an $A_{7}$ subsystem, while in $E_{8}$ an $\left(A_{1}^{4}\right)^{\prime}$ subsystem is one lying in an $A_{8}$ subsystem.)

\begin{tabular}{c|c|l}
$r$ & $d_{G_{2}, r}$ & $(p, Z)$ \\
\hline 1 & 14 & $\left(-, G_{2}\right)$ \\
2 & 6 & $\left(2, G_{2}\right),\left(-, A_{1} \tilde{A}_{1}\right)$ \\
3 & 4 & $\left(3, G_{2}\right),\left(-, \tilde{A}_{1} T_{1}\right)$ \\
4 & 4 & $\left(2, G_{2}\right),\left(-, A_{1} T_{1}\right)$ \\
5 & 4 & $\left(5, G_{2}\right),\left(-, A_{1} T_{1}\right)$
\end{tabular}

\begin{tabular}{r|c|l}
$r$ & $d_{F_{4}, r}$ & $(p, Z)$ \\
\hline 1 & 52 & $\left(-, F_{4}\right)$ \\
2 & 24 & $\left(2, F_{4}\right),\left(-, A_{1} C_{3}\right)$ \\
3 & 16 & $\left(3, F_{4}\right),\left(-, A_{2} \tilde{A}_{2}\right)$ \\
4 & 12 & $\left(2, F_{4}\right),\left(-, A_{1} \tilde{A}_{2} T_{1}\right)$ \\
5 & 12 & $\left(5, F_{4}\right),\left(-, A_{1} \tilde{A}_{2} T_{1}\right)$ \\
6 & 8 & $\left(2, A_{2} \tilde{A}_{2}\right),\left(3, A_{1} C_{3}\right),\left(-, A_{1} \tilde{A}_{1} T_{2}\right)$ \\
7 & 8 & $\left(7, F_{4}\right),\left(-, A_{1} \tilde{A}_{1} T_{2}\right)$ \\
8 & 6 & $\left(2, F_{4}\right),\left(-, \tilde{A}_{1} T_{3}\right)$ \\
9 & 6 & $\left(3, F_{4}\right),\left(-, \tilde{A}_{1} T_{3}\right)$ \\
10 & 6 & $\left(2, B_{2} T_{2}\right),\left(5, A_{1} C_{3}\right),\left(-, \tilde{A}_{1} T_{3}\right)$ \\
11 & 6 & $\left(11, F_{4}\right),\left(-, \tilde{A}_{1} T_{3}\right)$
\end{tabular}

\begin{tabular}{r|c|l}
$r$ & $d_{E_{6}, r}$ & $(p, Z)$ \\
\hline 1 & 78 & $\left(-, E_{6}\right)$ \\
2 & 38 & $\left(2, E_{6}\right),\left(-, A_{5} A_{1}\right)$ \\
3 & 24 & $\left(3, E_{6}\right),\left(-, A_{2}{ }^{3}\right)$ \\
4 & 20 & $\left(2, E_{6}\right),\left(-, A_{2}{ }^{2} A_{1} T_{1}\right)$ \\
5 & 16 & $\left(5, E_{6}\right),\left(-, A_{2} A_{1}^{2} T_{2}\right)$ \\
6 & 12 & $\left(2, A_{2}{ }^{3}\right),\left(3, A_{5} A_{1}\right),\left(-, A_{1}{ }^{3} T_{3}\right)$ \\
7 & 12 & $\left(7, E_{6}\right),\left(-, A_{1}{ }^{3} T_{3}\right)$ \\
8 & 10 & $\left(2, E_{6}\right),\left(-, A_{1}{ }^{2} T_{4}\right)$ \\
9 & 8 & $\left(3, E_{6}\right),\left(-, A_{1} T_{5}\right)$ \\
10 & 8 & $\left(2, A_{2} A_{1}{ }^{2} T_{2}\right),\left(5, A_{5} A_{1}\right),\left(-, A_{1} T_{5}\right)$ \\
11 & 8 & $\left(11, E_{6}\right),\left(-, A_{1} T_{5}\right)$
\end{tabular}




\begin{tabular}{r|c|l}
$r$ & $d_{E_{7}, r}$ & $(p, Z)$ \\
\hline 1 & 133 & $\left(-, E_{7}\right)$ \\
2 & 63 & $\left(2, E_{7}\right),\left(-, A_{7}\right)$ \\
3 & 43 & $\left(3, E_{7}\right),\left(-, A_{5} A_{2}\right)$ \\
4 & 33 & $\left(2, E_{7}\right),\left(-, A_{4} A_{2} T_{1}\right)$ \\
5 & 27 & $\left(5, E_{7}\right),\left(-, A_{3} A_{2} A_{1} T_{1}\right)$ \\
6 & 21 & $\left(2, A_{5} A_{2}\right),\left(3, A_{7}\right),\left(-, A_{2}{ }^{2} A_{1} T_{2}\right)$ \\
7 & 19 & $\left(7, E_{7}\right),\left(-, A_{2} A_{1}{ }^{3} T_{2}\right)$ \\
8 & 17 & $\left(2, E_{7}\right),\left(-, A_{2} A_{1}^{2} T_{3}\right)$ \\
9 & 15 & $\left(3, E_{7}\right),\left(-, A_{1}^{4} T_{3}\right)$ \\
10 & 13 & $\left(2, A_{3} A_{2} A_{1} T_{1}\right),\left(5, A_{7}\right),\left(-,\left(A_{1}{ }^{3}\right)^{\prime} T_{4}\right)$ \\
11 & 13 & $\left(11, E_{7}\right),\left(-,\left(A_{1}{ }^{3}\right)^{\prime} T_{4}\right)$ \\
12 & 11 & $\left(2, A_{5} A_{2}\right),\left(3, A_{3}{ }^{2} A_{1}\right),\left(-, A_{1}{ }^{2} T_{5}\right)$ \\
13 & 11 & $\left(13, E_{7}\right),\left(-, A_{1}{ }^{2} T_{5}\right)$ \\
14 & 9 & $\left(2, A_{2} A_{1}{ }^{3} T_{2}\right),\left(7, A_{7}\right),\left(-, A_{1} T_{6}\right)$ \\
15 & 9 & $\left(3, A_{3} A_{2} A_{1} T_{1}\right),\left(5, A_{5} A_{2}\right),\left(-, A_{1} T_{6}\right)$ \\
16 & 9 & $\left(2, E_{7}\right),\left(-, A_{1} T_{6}\right)$ \\
17 & 9 & $\left(17, E_{7}\right),\left(-, A_{1} T_{6}\right)$ \\
& &
\end{tabular}

\begin{tabular}{r|c|l}
$r$ & $d_{E_{8}, r}$ & $(p, Z)$ \\
\hline 1 & 248 & $\left(-, E_{8}\right)$ \\
2 & 120 & $\left(2, E_{8}\right),\left(-, D_{8}\right)$ \\
3 & 80 & $\left(3, E_{8}\right),\left(-, A_{8}\right)$ \\
4 & 60 & $\left(2, E_{8}\right),\left(-, D_{5} A_{3}\right)$ \\
5 & 48 & $\left(5, E_{8}\right),\left(-, A_{4}{ }^{2}\right)$ \\
6 & 40 & $\left(2, A_{8}\right),\left(3, D_{8}\right),\left(-, A_{4} A_{3} T_{1}\right)$ \\
7 & 36 & $\left(7, E_{8}\right),\left(-, A_{4} A_{2} A_{1}\right)$ \\
8 & 30 & $\left(2, E_{8}\right),\left(-, A_{3} A_{2} A_{1}{ }^{2} T_{1}\right)$ \\
9 & 28 & $\left(3, E_{8}\right),\left(-, A_{3} A_{2} A_{1} T_{2}\right)$ \\
10 & 24 & $\left(2, A_{4}{ }^{2}\right),\left(5, D_{8}\right),\left(-, A_{2}{ }^{2} A_{1}{ }^{2} T_{2}\right)$ \\
11 & 24 & $\left(11, E_{8}\right),\left(-, A_{2}{ }^{2} A_{1}^{2} T_{2}\right)$ \\
12 & 20 & $\left(2, A_{8}\right),\left(3, D_{5} A_{3}\right),\left(-, A_{2} A_{1}{ }^{3} T_{3}\right)$ \\
13 & 20 & $\left(13, E_{8}\right),\left(-, A_{2} A_{1}{ }^{3} T_{3}\right)$ \\
14 & 18 & $\left(2, A_{4} A_{2} A_{1}\right),\left(7, D_{8}\right),\left(-, A_{2} A_{1}^{2} T_{4}\right)$ \\
15 & 16 & $\left(3, A_{4}{ }^{2}\right),\left(5, A_{8}\right),\left(-,\left(A_{1}^{4}\right)^{\prime} T_{4}\right)$ \\
16 & 16 & $\left(2, E_{8}\right),\left(-,\left(A_{1}^{4}\right)^{\prime} T_{4}\right)$ \\
17 & 16 & $\left(17, E_{8}\right),\left(-,\left(A_{1}{ }^{4}\right)^{\prime} T_{4}\right)$ \\
18 & 14 & $\left(2, A_{3} A_{2} A_{1} T_{2}\right),\left(3, D_{8}\right),\left(-, A_{1}{ }^{3} T_{5}\right)$ \\
19 & 14 & $\left(19, E_{8}\right),\left(-, A_{1}{ }^{3} T_{5}\right)$ \\
20 & 12 & $\left(2, A_{4}{ }^{2}\right),\left(5, D_{5} A_{3}\right),\left(-, A_{1}{ }^{2} T_{6}\right)$ \\
21 & 12 & $\left(3, A_{4} A_{2} A_{1} T_{1}\right),\left(7, A_{8}\right),\left(-, A_{1}{ }^{2} T_{6}\right)$ \\
22 & 12 & $\left(2, A_{2}{ }^{2} A_{1}{ }^{2} T_{2}\right),\left(11, D_{8}\right),\left(-, A_{1}{ }^{2} T_{6}\right)$ \\
23 & 12 & $\left(23, E_{8}\right),\left(-, A_{1}^{2} T_{6}\right)$ \\
24 & 10 & $\left(2, A_{8}\right),\left(3, A_{3} A_{2} A_{1}^{2} T_{1}\right),\left(-, A_{1} T_{7}\right)$ \\
25 & 10 & $\left(5, E_{8}\right),\left(-, A_{1} T_{7}\right)$ \\
26 & 10 & $\left(2, A_{2} A_{1}{ }^{3} T_{3}\right),\left(13, D_{8}\right),\left(-, A_{1} T_{7}\right)$ \\
27 & 10 & $\left(3, E_{8}\right),\left(-, A_{1} T_{7}\right)$ \\
28 & 10 & $\left(2, A_{4} A_{2} A_{1} T_{1}\right),\left(7, D_{5} A_{3}\right),\left(-, A_{1} T_{7}\right)$ \\
29 & 10 & $\left(29, E_{8}\right),\left(-, A_{1} T_{7}\right)$ \\
& &
\end{tabular}

In all cases we see that $d_{q}(Z)=d_{\Phi, r}$, as required. This completes the proof in the cases where $r<h$. 
Now assume $r \geq h$; here we have $d_{\Phi, r}=\ell$, so we seek to show that there are regular elements in $G_{[r]}$. As we are assuming $n<h$, we may consider the $n$th row of the relevant table above, and take $Z$ to be the group appearing in the final entry of the row (so that $Z$ is the centralizer of a semisimple element of order $n$, provided the characteristic does not divide $n$ ). We then wish to show that there are regular unipotent elements in $Z_{[q]}$. To see this we set $h_{Z}$ to be the maximum of the Coxeter numbers of the simple factors of $Z$, and observe that in almost all cases we have $h_{Z}=\left\lceil\frac{h}{n}\right\rceil ;$ the exceptions are listed in the table below:

\begin{tabular}{c|c|c|c|c}
$G$ & $n$ & $Z$ & $h_{Z}$ & $\frac{h}{n}$ \\
\hline$G_{2}$ & 2 & $A_{1} \tilde{A}_{1}$ & 2 & 3 \\
$F_{4}$ & 3 & $A_{2} \tilde{A}_{2}$ & 3 & 4 \\
$E_{6}$ & 3 & $A_{2}{ }^{3}$ & 3 & 4 \\
$E_{7}$ & 2 & $A_{7}$ & 8 & 9 \\
$E_{8}$ & 2 & $D_{8}$ & 14 & 15 \\
$E_{8}$ & 3 & $A_{8}$ & 9 & 10 \\
$E_{8}$ & 5 & $A_{4}{ }^{2}$ & 5 & 6
\end{tabular}

In each of these exceptions we have $h_{Z}=\frac{h}{n}-1$; and we observe that in each of them $h_{Z}$ fails to be coprime to $n$ and so cannot be a power of $p$. Thus in all instances the conditions $p^{a} \geq h_{Z}$ and $p^{a} \geq \frac{h}{n}$ are equivalent. We now employ Testerman's order formula (which is proved in [21] for good characteristic, but may easily be verified in bad characteristic in the case of regular unipotent elements): this states that the order of the regular unipotent elements of $Z$ is $\min \left\{p^{a}: p^{a} \geq h_{Z}\right\}$, which we may now write as $\min \left\{p^{a}: p^{a} \geq \frac{h}{n}\right\}$. Since by assumption $q \geq \frac{h}{n}$, it follows that $Z_{[q]}$ does indeed contain regular unipotent elements as required. This completes the proof in the cases where $r \geq h$.

Combining Lemma [3.1] with Propositions [3.3, [3.4, [3.5, [3.6] and [3.7] we have proved the following.

Theorem 3.8. If $G$ is a simple algebraic group of adjoint type with root system $\Phi$, and $r \in \mathbb{N}$, then $\operatorname{codim} G_{[r]}=d_{\Phi, r}$.

At this point we may observe that taking this result together with Lemma 1.2 gives the following immediate consequence concerning dimensions rather than codimensions.

Corollary 3.9. If $G$ is a simple algebraic group of adjoint type with root system $\Phi$, and $r \in \mathbb{N}$, set $x=\left(1-\frac{1}{r}\right) \operatorname{dim} G-\operatorname{dim} G_{[r]}$. Then $x \geq 0$ if $r$ is a product of very good primes, and if $G$ is of classical type, the value of $x$ is given by

$$
x= \begin{cases}\frac{1}{r}(e-1)(r-e-1) & \text { if } \Phi=A_{\ell}, \\ \frac{1}{2 r} e(r-e-1)+\left(\epsilon_{r}\left\lceil\frac{z}{2}\right\rceil-\frac{z}{2}\right) & \text { if } \Phi=B_{\ell} \text { or } C_{\ell}, \\ \frac{1}{2 r}(e+2)(r-e-1)+\left(\epsilon_{r}\left\lceil\frac{z}{2}\right\rceil-\frac{z}{2}-\epsilon_{z}\right) & \text { if } \Phi=D_{\ell} .\end{cases}
$$

We may now prove our result concerning $G_{(r)}$.

Theorem 3.10. If $G$ is a simple algebraic group of adjoint type with root system $\Phi$, and $G_{(r)} \neq \emptyset$ for some $r \in \mathbb{N}$, then $\operatorname{codim} G_{(r)}=d_{\Phi, r}$.

Proof. Given $G$, suppose $r$ is a minimal counterexample to the statement to be proved. Then $G_{[r]}$ contains elements of order $r$, but the minimal centralizer dimension occurs for elements of order $r^{\prime}$ for some $r^{\prime}<r$. Write as usual $r=n q$. 
The calculations in Propositions 3.3, 3.4, 3.5, 3.6 and 3.7 provide a centralizer $Z$ of a semisimple element of order $n$ with the required value of $d_{q}(Z)$, so we must have $r^{\prime}=n q^{\prime}$ for some $q^{\prime}<q$. Since elements of order $r^{\prime}$ therefore lie in $G_{[r / p]}$, we must have $\operatorname{codim} G_{[r / p]}=d_{\Phi, r}$, i.e., $d_{\Phi, r / p}=d_{\Phi, r}$; by Lemma 1.3 this forces $d_{\Phi, r / p}=\ell$ (and thus $r / p \geq h$ ). Note that the minimality of $r$, and the fact that $G_{(r / p)}$ contains the $p$ th powers of the elements in $G_{(r)}$ and thus is non-empty, mean that $\operatorname{codim} G_{(r / p)}=\ell$; so we may assume that $r^{\prime}=r / p$.

Let

$$
H_{n}=\bigcup_{s \in G_{(n)}}\left\{u \in G: u \text { a regular unipotent element of } C_{G}(s)^{0}\right\},
$$

and let $q^{\prime \prime}=\max _{u \in H_{n}} o(u)$. Since regular unipotent elements in any algebraic group have the maximal orders among the unipotent elements there, we must have $q^{\prime \prime} \geq q$. If we had $q^{\prime \prime}=q$ we would have regular elements of order $r$ as required, so we must have $q^{\prime \prime}>q$. Thus on the one hand we have regular elements of order $r p^{x}$, where $q^{\prime \prime}=q p^{x}$, and on the other we have regular elements of order $r^{\prime}=r / p$. We shall show that there must then be regular elements of order $r$, contrary to the choice of $r$.

Our approach for $G$ of classical type is to begin with a regular element $g=s u$ of order $r^{\prime}$ and successively change the element while maintaining the order of its semisimple part. Thus if $\Phi$ is of type $A_{\ell}$, the centralizer $Z$ of $s$ is a product of factors of type $A$ and a torus. If the largest type $A$ factor has rank $k$, we must have $q^{\prime} / p \leq k<q^{\prime}$, because the order of a regular unipotent element in $A_{k}$ is the smallest power of $p$ which is greater than $k$. Now if $A_{k^{\prime}}$ is any other type $A$ factor, replace $A_{k} A_{k^{\prime}}$ by $A_{k+1} A_{k^{\prime}-1}$ and iterate until $Z$ has just one non-trivial type $A$ factor; then iterate replacing $A_{\ell-y} T_{y}$ by $A_{\ell-y+1} T_{y-1}$ until $A_{\ell-1} T_{1}$ is reached. (Note that each such change still leaves a centralizer of a semisimple element of order $n$. In terms of the result from [6], we may take all but one of the non-zero coefficients $b_{i}$ to be 1 , and the last to be determined by the requirement that $\sum b_{i}=n$.) At each stage the order of regular unipotent elements can change by at most a factor of $p$. Since the order begins at $q^{\prime}=q / p$ and finishes at $q^{\prime \prime}=q p^{x}$, after the first increase we must have regular unipotent elements of order $q$ and therefore regular elements of order $r$ as required.

The argument for the other classical groups proceeds in like fashion. If $\Phi$ is of type $C_{\ell}$, the centralizer $Z$ has factors of type $A$, at most two factors of type $C$ and a torus. If there is no type $C$ factor, begin by replacing some $A_{k}$ by $C_{k}$. Iterate replacing $C_{k} A_{k^{\prime}}$ by $C_{k+1} A_{k^{\prime}-1}$ until there are no non-trivial type $A$ factors remaining. If there are two type $C$ factors, iterate replacing $C_{k} C_{k^{\prime}}$ (where $k \geq k^{\prime}$ ) by $C_{k+1} C_{k^{\prime}-1}$ until there is only one. Finally iterate replacing $C_{\ell-y} T_{y}$ by $C_{\ell-y+1} T_{y-1}$ until $C_{\ell-1} T_{1}$ is reached. Again, at each stage the order of regular unipotent elements can change by at most a factor of $p$, so the result follows. Types $D_{\ell}$ and $B_{\ell}$ are precisely similar.

For $G$ of exceptional type we proceed differently, as it is necessary to be more careful about the coefficients $b_{i}$ which determine the centralizer $Z$. Here we simply form a list of all possible centralizers $Z$, and for each record the possible values of $n_{1}$ for which it is the centralizer of a semisimple element of order $n_{1}$, together with the order $q_{1}$ of regular unipotent elements in $Z$. This therefore lists all possible pairs $\left(n_{1}, q_{1}\right)$ for which there is a regular element of order $n_{1} q_{1}$. Thus if there are elements of order $n_{1}{ }^{\prime} q_{1}{ }^{\prime}$, then there is a pair $\left(n_{1}, q_{1}\right)$ on the list with $n_{1}{ }^{\prime}=n_{1}$ 
and $q_{1}{ }^{\prime} \leq q_{1}$. We next delete any lines for which the possible pairs $\left(n_{1}, q_{1}\right)$ form a subset of those on some other line. (For example, if $\Phi$ is of type $E_{8}$, then the pairs $\left(n_{1}, q_{1}\right)$ corresponding to $Z=A_{1} T_{7}$ are those with $n_{1} \geq 24$ and $q_{1}=p$, while those corresponding to $Z=A_{1}^{2} T_{6}$ are those with $n_{1} \geq 20$ and $q_{1}=p$; the first line may be deleted as each of its pairs occurs in the second.) We then form a second list, entering opposite each remaining $Z$ all pairs $\left(n_{1}, q_{1}{ }^{\prime}\right)$ satisfying $n_{1} q_{1}{ }^{\prime} \geq h$ for which there is a pair $\left(n_{1}, q_{1}\right)$ on the first list with $q_{1}{ }^{\prime}<q_{1}$. For example, the result for $\Phi$ of type $E_{6}$ is as follows, in which the values of $q_{1}{ }^{\prime}$ in the final column correspond to values of $n_{1}$ for which $n_{1} q_{1}{ }^{\prime} \geq 12$ :

\begin{tabular}{c|c|l|l}
$Z$ & $n_{1}$ & $q_{1}$ & $q_{1}{ }^{\prime}$ \\
\hline$T_{6}$ & $\geq 12$ & 1 & - \\
$A_{1}{ }^{3} T_{3}$ & $\geq 6$ & $p$ & 1 \\
$A_{3} A_{1} T_{2}$ & $\geq 4$ & $4,9, p>3$ & $1,2,3$ \\
$A_{2}{ }^{2} A_{1} T_{1}$ & $\geq 4$ & $4, p>2$ & 1,2 \\
$A_{4} A_{1} T_{1}$ & $\geq 3$ & $8,9, p>3$ & $1,2,3,4$ \\
$A_{5} T_{1}$ & $\geq 3$ & $8,9,25, p>5$ & $1,2,3,4,5$ \\
$D_{5} T_{1}$ & $\geq 2$ & $8,9,25,49, p>7$ & $1,2,3,4,5,7$ \\
$A_{2}{ }^{3}$ & 3 & $4, p>3$ & - \\
$A_{5} A_{1}$ & 2 & $9,25, p>5$ & - \\
$E_{6}$ & 1 & $16,27,25,49,121, p>11$ & -
\end{tabular}

By inspection we find that each pair on the second list occurs on the first list opposite some other possibility for $Z$ : those with $q_{1}^{\prime}=1$ occur for $Z=T_{6}$ (for $n \geq 12$ ); those with $q_{1}{ }^{\prime}=2$ for $Z=A_{1}{ }^{3} T_{3}$ (for $n \geq 6$ ); those with $q_{1}{ }^{\prime}=3$ for $Z=A_{2}{ }^{2} A_{1} T_{1}$ (for $n \geq 4$ ); those with $q_{1}{ }^{\prime}=4$ for $Z=A_{2}{ }^{3}$ (for $n=3$ ) or $Z=A_{2}^{2} A_{1} T_{1}$ (for $n \geq 4$ ); those with $q_{1}^{\prime}=5$ for $Z=A_{4} A_{1} T_{1}$ (for $n \geq 3$ ); and those with $q_{1}{ }^{\prime}=7$ for $Z=A_{5} A_{1}$ (for $n=2$ ) or $Z=A_{5} T_{1}$ (for $n \geq 3$ ). This happens for each exceptional group $G$. Thus if there are elements in $G$ of a given order greater than or equal to $h$, then there are regular elements of that order. Since by the above it suffices to consider regular elements, this proves the result.

Finally we consider groups of arbitrary isogeny type.

Theorem 3.11. If $G$ is a simple algebraic group with root system $\Phi$, and $r \in \mathbb{N}$, then $\operatorname{codim} G_{[r]} \geq d_{\Phi, r}$.

Proof. Let $G_{\mathrm{ad}}$ be the adjoint group of the same type and over the same field as $G$, and let $\phi: G \rightarrow G_{\text {ad }}$ be an isogeny; for $x \in G$ write $\hat{x}$ for $\phi(x)$. Take $g \in G_{[r]}$; then $\hat{g} \in\left(G_{\mathrm{ad}}\right)_{[r]}$. Given $h \in G$, the set $G_{g, h}=\{x \in G:[g, x]=h\}$ is either empty or a right coset of $C_{G}(g)$. As $C_{G_{\text {ad }}}(\hat{g})=\bigcup_{h \in \operatorname{ker} \phi} \phi\left(G_{g, h}\right)$ and $\operatorname{ker} \phi$ is finite, Theorem 3.8 gives $\operatorname{dim} C_{G}(g)=\operatorname{dim} C_{G_{\text {ad }}}(\hat{g}) \geq d_{\Phi, r}$. By Lemma 3.1 we then have $\operatorname{codim} G_{[r]}=d_{r}(G) \geq d_{\Phi, r}$, as required.

Note that an inequality is the best possible result here, as may be seen by considering groups of type $A_{1}$ in odd characteristic with $r=2$. We have $d_{A_{1}, 2}=1$ : if $G$ is the adjoint group $P G L_{2}(K)$, the involution which is the image of $\operatorname{diag}(1,-1)$ is a regular semisimple element, giving codim $G_{[2]}=1$ as required by Theorem 3.8 However, if $G$ is the simply-connected group $S L_{2}(K)$, the only involution in $G$ is the central element $\operatorname{diag}(-1,-1)$, so that $\operatorname{codim} G_{[2]}=3$.

Combining Lemma [1.2, Theorem 3.8 and Theorem 3.11 completes the proof of Theorem 1 


\section{REFERENCES}

[1] N. Bourbaki, Groupes et algèbres de Lie, Chapitres 4, 5 and 6, Hermann, Paris (1975). MR.0240238 (39:1590)

[2] J.F. Carlson, Z. Lin, D.K. Nakano and B.J. Parshall, "The restricted nullcone", Combinatorial and Geometric Representation Theory (Seoul, 2001), Contemp. Math. 325 (2003), 51-75. MR 1988985 (2004g:17017)

[3] B. Chang, "The conjugate classes of Chevalley groups of type $\left(G_{2}\right)$ ", J. Algebra 9 (1968), 190-211. MR 0227258 (37:2843)

[4] D. Deriziotis, "Centralizers of semisimple elements in a Chevalley group", Comm. Algebra 9 (1981), 1997-2014. MR0638635|(83e:20043)

[5] H. Enomoto, "The conjugacy classes of Chevalley groups of type $\left(G_{2}\right)$ over finite fields of characteristic 2 or 3", J. Fac. Sci. Univ. Tokyo 16 (1970), 497-512. MR0262352 (41:6960)

[6] B. Hartley and M. Kuzucuoğlu, "Centralizers of elements in locally finite simple groups", Proc. London Math. Soc. 62 (1991), 301-324. MF,1085643 (92d:20040)

[7] W.H. Hesselink, "Nilpotency in classical groups over a field of characteristic 2", Math. Z. 166 (1979), 165-181. MR0525621 (82d:14030)

[8] R. Lawther, "Jordan block sizes of unipotent elements in exceptional algebraic groups", Comm. Algebra 23(11) (1995), 4125-4156. MR1351124 (96h:20084)

[9] R. Lawther, M.W. Liebeck and G.M. Seitz, "Fixed point spaces in actions of exceptional algebraic groups", Pac. J. Math. 205 (2002), 339-391. MF.1922739 (2003g:20080)

[10] M.W. Liebeck and A. Shalev, "Classical groups, probabilistic methods and the (2,3)generation problem", Annals of Math. 144 (1996), 77-125. MF 1405944 (97e:20106a)

[11] M.W. Liebeck and A. Shalev, "Random $(r, s)$-generation of finite classical groups", Bull. London Math. Soc. 34 (2002), 185-188. MR1874245 (2002i:20100)

[12] M.W. Liebeck and A. Shalev, "Fuchsian groups, finite simple groups and representation varieties", to appear in Invent. Math.

[13] T. Matsuki, "The orbits of affine symmetric spaces under the action of minimal parabolic subgroups", J. Math. Soc. Japan 31 (1979), 331-357. MR.0527548 (81a:53049)

[14] K. Mizuno, "The conjugate classes of Chevalley groups of type $E_{6}$ ", J. Fac. Sci. Univ. Tokyo 24 (1977), 525-563. MR0486170 (58:5951)

[15] K. Mizuno, "The conjugate classes of unipotent elements of the Chevalley groups $E_{7}$ and E8", Tokyo J. Math 3 (1980), 391-461. MR0605099 (82m:20046)

[16] G.M. Seitz, "Double cosets in algebraic groups", in Algebraic groups and their representations (ed. R.W. Carter and J. Saxl), NATO ASI Series 517, Kluwer, Dordrecht (1998), pp. 241-257. MR $1670773(99 \mathrm{k}: 20096)$

[17] K. Shinoda, "The conjugacy classes of Chevalley groups of type $\left(F_{4}\right)$ over finite fields of characteristic 2", J. Fac. Sci. Univ. Tokyo 21 (1974), 133-159. MR0349863 (50:2356)

[18] T. Shoji, "The conjugacy classes of Chevalley groups of type $\left(F_{4}\right)$ over finite fields of characteristic $p \neq 2$ ", J. Fac. Sci. Univ. Tokyo 21 (1974), 1-17. MR0357641 (50:10109)

[19] T.A. Springer, "Some results on algebraic groups with involutions", in Algebraic groups and related topics (ed. R. Hotta), Advanced Studies in Pure Mathematics 6, Kinokuniya, Tokyo and North-Holland, Amsterdam (1985), pp. 525-543. MR0803346 (86m:20050)

[20] T.A. Springer and R. Steinberg, "Conjugacy classes", in Seminar on algebraic groups and related finite groups (ed. A. Borel et al.), Lecture Notes in Mathematics 131, Springer, Berlin (1970), pp. 167-266. MR0268192(42:3091)

[21] D.M. Testerman, " $A_{1}$-type overgroups of elements of order $p$ in semisimple algebraic groups and the associated finite groups", J. Algebra 177 (1995), 34-76. MR1356359 (96j:20067)

Department of Mathematics and Statistics, Lancaster University, Lancaster LA1 4YF, United Kingdom

Current address: Department of Pure Mathematics and Mathematical Statistics, Centre for Mathematical Sciences, Cambridge University, Cambridge CB3 0WB, United Kingdom 